\title{
Filter descriptive classes of Borel functions
}

by

\author{
Gabriel Debs and Jean Saint Raymond (Paris)
}

\begin{abstract}
We first prove that given any analytic filter $\mathcal{F}$ on $\omega$ the set of all functions $f$ on $\mathbf{2}^{\omega}$ which can be represented as the pointwise limit relative to $\mathcal{F}$ of some sequence $\left(f_{n}\right)_{n \in \omega}$ of continuous functions $\left(f=\lim _{\mathcal{F}} f_{n}\right)$, is exactly the set of all Borel functions of class $\xi$ for some countable ordinal $\xi$ that we call the rank of $\mathcal{F}$. We discuss several structural properties of this rank. For example, we prove that any free $\boldsymbol{\Pi}_{4}^{0}$ filter is of rank 1.
\end{abstract}

The idea of associating to a given filter $\mathcal{F}$ a class of functions is due to Katětov (see [5] and [6]). In this work we restrict our study to classes of Borel functions on Polish spaces and we shall assume the reader to be familiar with Descriptive Set Theory in this context. Nevertheless to avoid any ambiguity we shall recall explicitly in this introduction a number of elementary notions.

Descriptive pointclasses. Informally speaking, a descriptive pointclass $\boldsymbol{\Gamma}$ is a general assignment to any Polish space $X$ of a family $\boldsymbol{\Gamma}(X)$ of subsets of $X$ with the property that for any continuous mapping $\varphi: X \rightarrow Y$ between two Polish spaces $X$ and $Y$, if $A \in \boldsymbol{\Gamma}(Y)$ then $\varphi^{-1}(A) \in \boldsymbol{\Gamma}(X)$. More formally, $\boldsymbol{\Gamma}$ should be viewed as a formula in the language of Set Theory, and $\boldsymbol{\Gamma}(X)$ the set obtained by relativization of this formula to the structure induced by the Polish topology on $X$. Still we shall very often refer to $\Gamma(X)$ as a "class of sets on $X$ ". Typical examples of such classes are the (additive, multiplicative, ambiguous) Borel classes $\boldsymbol{\Sigma}_{\xi}^{0}, \boldsymbol{\Pi}_{\xi}^{0}, \boldsymbol{\Delta}_{\xi}^{0}$ or the classes $\boldsymbol{\Delta}_{1}^{1}, \boldsymbol{\Sigma}_{1}^{1}$, $\Pi_{1}^{1}$ (of Borel, analytic, coanalytic sets).

Throughout, by a function we shall always mean a real-valued mapping.

Descriptive classes of functions. In the same way, a descriptive class $\mathcal{D}$ of functions is a general assignment to any Polish space $X$ of a family $\mathcal{D}(X)$

2000 Mathematics Subject Classification: Primary 03E15; Secondary 03E45, 54A20, 54H05.

Key words and phrases: analytic filters, Borel functions. 
of functions on $X$ with the property that for any continuous mapping $\varphi$ : $X \rightarrow Y$ between two Polish spaces $X$ and $Y$, if $f \in \mathcal{D}(Y)$ then $\varphi \circ f \in \mathcal{D}(X)$. We shall also refer to $\mathcal{D}(X)$ as a "class" of functions.

In this work we shall mainly be interested in the class $\mathcal{B}$ of all Borel functions, and for any countable ordinal $\xi$, in the Borel class $\mathcal{B}_{\xi}$ and the Baire class $\mathcal{B}^{(\xi)}$. We next briefly recall these notions.

The Borel classes $\mathcal{B}_{\xi}$. For any Polish space $X, \mathcal{B}_{\xi}(X)$ is the set of all functions $f: X \rightarrow \mathbb{R}$ such that the inverse image of any open subset of $\mathbb{R}$ is a $\boldsymbol{\Sigma}_{1+\xi}^{0}$ subset of $X$. In particular, $\mathcal{B}_{0}(X)$ is just the set of all continuous functions on $X$.

The Baire classes $\mathcal{B}^{(\xi)}$. For any Polish space $X$ the sets $\mathcal{B}^{(\xi)}(X)$ are defined inductively: $\mathcal{B}^{(0)}(X)$ is the set of all continuous functions on $X$, and for any countable ordinal $\xi>0, \mathcal{B}^{(\xi)}(X)$ is the set of all pointwise limits of sequences from $\bigcup_{\eta<\xi} \mathcal{B}^{(\eta)}(X)$.

We recall that (in the context of functions of real variables) the classes $\mathcal{B}^{(\xi)}$ were introduced first, by Baire in his thesis [1], while the Borel classes (of successor rank, $\mathcal{B}_{\xi+1}$ ) were introduced later on by Lebesgue in his famous memoir [8] where he also proved the fundamental result that

$$
\mathcal{B}^{(1+\xi)}(X)=\mathcal{B}_{\xi+1}(X) \text {. }
$$

All these results were then generalized by Hausdorff to arbitrary Polish spaces. So the Baire classes are just the Borel classes of successor rank.

We now come to the central concept of "filter descriptive class of functions". This notion was initially introduced by Katětov for filters with arbitrary domain. However, all filters we shall consider in this work will be with countable domain, and unless otherwise specified, "filter" will mean "filter on $\omega$ ". As usual, when we speak about the descriptive complexity of such a filter $\mathcal{F}$, we view $\mathcal{F}$ as a subset of the Cantor space via the canonical identification $\mathcal{P}(\omega) \approx \mathbf{2}^{\omega}$.

Definition (Katětov). Let $\mathcal{F}$ be a filter. For any topological space $X$ we denote by $\mathcal{C}_{\mathcal{F}}(X)$ the set of all functions $f$ on $X$ which can be represented

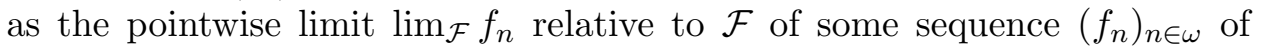
continuous functions on $X$.

A class $\mathcal{D}$ of functions is said to be a filter descriptive class if there exists a filter $\mathcal{F}$ such that for any Polish space $X, \mathcal{D}(X)=\mathcal{C}_{\mathcal{F}}(X)$. The class $\mathcal{D}$ is then said to be generated by the filter $\mathcal{F}$.

The main examples of filter descriptive classes are the Borel classes. More precisely:

Any Borel class $\mathcal{B}_{\xi}$ of functions is generated by a Borel filter. 
This was proved by Katětov for Baire classes, that is, when $\xi$ is successor, but as we shall see it is also true for $\xi$ limit. This additional information is of some importance since our first goal is to prove the following converse.

Theorem A. For any $\boldsymbol{\Sigma}_{1}^{1}$ filter $\mathcal{F}$ there exists a countable ordinal $\xi$ (called the rank of $\mathcal{F}$ ) such that for any zero-dimensional Polish space $X$, $\mathcal{C}_{\mathcal{F}}(X)$ is precisely the Borel class $\mathcal{B}_{\xi}(X)$.

We conjecture that in Theorem A the zero-dimensionality assumption on $X$ can be dropped, and we shall prove that this is indeed the case when $\xi \leq 2$. But we point out that the $\boldsymbol{\Sigma}_{1}^{1}$ assumption on the filter $\mathcal{F}$ cannot be released since by Theorem 2 of [9]:

(Louveau) The class $\mathcal{B}$ of all Borel functions is generated by a $\Pi_{1}^{1}$ filter.

In fact, Theorem A will be derived from the combination of two more general results, characterizing each of the two inclusions: $\mathcal{C}_{\mathcal{F}}(X) \subset \mathcal{B}_{\xi}(X)$ and $\mathcal{C}_{\mathcal{F}}(X) \supset \mathcal{B}_{\xi}(X)$, from which we shall also derive the following:

Theorem B. Any $\boldsymbol{\Pi}_{\xi}^{0}$ free filter $\mathcal{F}$ is separated from its dual ideal by a $\Sigma_{\eta}^{0}$ set for some $\eta<\xi$.

This latter result answers a question asked by Solecki in [15] where he proved the particular case $\xi=3$. It is interesting to point out that Solecki's motivation for considering these separation conditions was far away from ours and was actually dictated by some complexity computation (see Remark 2.11 below).

We shall also study several closure properties of this rank and some canonical constructions of filters of arbitrary rank.

In the last part of the paper we shall discuss two conjectures on the structure of $\boldsymbol{\Sigma}_{1}^{1}$ filters of rank $\geq \xi$, which we shall prove for $\xi \leq 2$. In particular, we obtain the following two results:

THEOREM C. There exists a $\boldsymbol{\Sigma}_{4}^{0}$ filter of rank 2 which embeds in any $\boldsymbol{\Sigma}_{1}^{1}$ filter of rank $\geq 2$.

Here by "embedding" a filter $\mathcal{F}$ in a filter $\mathcal{G}$ we mean that up to a bijection between their domains, $\mathcal{F}$ is coarser than $\mathcal{G}$.

Theorem D. Any $\boldsymbol{\Pi}_{4}^{0}$ filter is of rank $\leq 1$.

\section{Preliminaries}

1.1. The canonical involution on $\mathcal{P}(E)$. Given any set $E$ the canonical involution $\sigma: A \mapsto E \backslash A$ on $\mathcal{P}(E)$ will play a fundamental role in our study. Also given any subset $\mathcal{A}$ of $\mathcal{P}(E)$ we define

$$
\mathcal{A}^{*}:=\sigma(\mathcal{A})=\{E \backslash A: A \in \mathcal{A}\} .
$$


Observe that since $\sigma$ is a homeomorphism, $\mathcal{A}$ is in some pointclass $\boldsymbol{\Gamma}$ if and only if $\mathcal{A}^{*}$ is in $\boldsymbol{\Gamma}$. If $\mathcal{F}$ is a filter on $E$ then $\mathcal{F}^{*}$ is its associated ideal.

1.2. Separation properties. Let $\boldsymbol{\Gamma}$ be a pointclass, and $A$ and $B$ two subsets of a Polish space $X$. We shall say that $A$ is $\boldsymbol{\Gamma}$-separated from $B$ if there exists a subset $S$ of $X$ in $\boldsymbol{\Gamma}$ such that $A \subset S$ and $S \cap B=\emptyset$.

As we shall see, behind the problem under study lies a separation problem between the given filter $\mathcal{F}$ and its associated ideal $\mathcal{F}^{*}$. But before going into further details let us point out two general elementary properties.

Lemma 1.3. For any $\mathcal{A} \subset \mathcal{P}(\omega)$ the following are equivalent:

(i) $\mathcal{A}$ is $\boldsymbol{\Sigma}_{\xi}^{0}$-separated from $\mathcal{A}^{*}$.

(ii) $\mathcal{A}$ is $\Pi_{\xi}^{0}$-separated from $\mathcal{A}^{*}$.

(iii) $\mathcal{A}$ and $\mathcal{A}^{*}$ are contained in two disjoint $\boldsymbol{\Sigma}_{\xi}^{0}$ sets.

Proof. For the equivalence (i) $\Leftrightarrow$ (ii) observe that if $\mathcal{B}$ separates $\mathcal{A}$ from $\mathcal{A}^{*}$ then $\mathcal{P}(\omega) \backslash \mathcal{B}^{*}$ also separates $\mathcal{A}$ from $\mathcal{A}^{*}$.

The implication (i) $\Rightarrow$ (iii) follows from the reduction property of the class $\boldsymbol{\Sigma}_{\xi}^{0}$, and the converse is obvious.

Lemma 1.4. For any $\mathcal{A} \subset \mathcal{P}(\omega)$ the following are equivalent:

(i) $\mathcal{A}$ is $\boldsymbol{\Delta}_{\xi}^{0}$-separated from $\mathcal{A}^{*}$.

(ii) $\mathcal{A}$ and $\mathcal{A}^{*}$ are contained in two disjoint $\mathbf{\Pi}_{\xi}^{0}$ sets.

Proof. If $\mathcal{B}$ is a $\boldsymbol{\Delta}_{\xi}^{0}$ set separating $\mathcal{A}$ from $\mathcal{A}^{*}$, then $\mathcal{B}$ and $\mathcal{B}^{*}$ are disjoint $\Pi_{\xi}^{0}$ sets containing $\mathcal{A}$ and $\mathcal{A}^{*}$. Conversely, if $\mathcal{B}_{0}$ and $\mathcal{B}_{1}$ are two disjoint $\boldsymbol{\Pi}_{\xi}^{0}$ sets containing $\mathcal{A}$ and $\mathcal{A}^{*}$, it follows from the separation property of the class $\boldsymbol{\Pi}_{\xi}^{0}$ that there exists a $\boldsymbol{\Delta}_{\xi}^{0}$ set $\mathcal{B}$ separating $\mathcal{B}_{0}$ from $\mathcal{B}_{1}$, hence $\mathcal{A}$ from $\mathcal{A}^{*}$.

2. Filter descriptive classes versus Borel classes. Our goal in this section is to characterize the inclusions $\mathcal{C}_{\mathcal{F}}(X) \subset \mathcal{B}_{\xi}(X)$ and $\mathcal{C}_{\mathcal{F}}(X) \supset$ $\mathcal{B}_{\xi}(X)$. For this we need to prove a number of closure properties of the set $\mathcal{C}_{\mathcal{F}}(X)$.

In all that follows, $X$ denotes a Polish space, $\Gamma$ a pointclass, $\mathcal{F}$ a filter on $\omega$, and $\xi$ a countable ordinal. When not otherwise specified, limits in $\mathbb{R}^{X}$ are relative to the pointwise convergence (product) topology.

Whenever $A$ is a subset of $X, \mathbb{1}_{A}: X \rightarrow\{0,1\}$ will denote its characteristic function. And if $f$ is a real-valued function on $X$ and $t \in \mathbb{R}$, we will denote by $[f \leq t]$ (resp. $[f \geq t]$ ) the set $\{x \in X: f(x) \leq t\}$ (resp. $\{x \in X: f(x) \geq t\})$.

Lemma 2.1. If $\mathbb{1}_{A} \in \mathcal{C}_{\mathcal{F}}(X)$ for all $A \in \boldsymbol{\Delta}_{1+\xi}^{0}$ then $\mathcal{B}_{\xi}(X) \subset \mathcal{C}_{\mathcal{F}}(X)$.

Proof. Let $f \in \mathcal{B}_{\xi}(X)$; we have to show that $f \in \mathcal{C}_{\mathcal{F}}(X)$. 
Consider first the particular case where $f$ is valued in the unit interval $[0,1]$ and fix $1 / 3<s<t<2 / 3$. Starting from $f_{0}=f$ define inductively, for all $n \in \omega, f_{n} \in \mathcal{B}_{\xi}$ and $A_{n} \in \Delta_{1+\xi}^{0}$ such that $A_{n}$ separates $\left[f_{n} \leq s\right]$ from $\left[f_{n} \geq t\right]$ and $f_{n+1}=\frac{3}{2}\left(f_{n}-\frac{1}{3} \mathbb{1}_{A_{n}}\right)$. This construction is possible since the disjoint sets $\left[f_{n} \leq s\right]$ and $\left[f_{n} \geq t\right]$ are in $\Pi_{1+\xi}^{0}$. Then one easily checks that $f=\sum_{n=0}^{\infty} \alpha_{n} \mathbb{1}_{A_{n}}$ with $\alpha_{n}=2^{n} / 3^{n+1}$. Now since each $\mathbb{1}_{A_{n}} \in \mathcal{C}_{\mathcal{F}}(X)$ we can fix a sequence $\left(g_{\langle n, k\rangle}\right)_{k \in \omega}$ of continuous functions on $X$ such that $\mathbb{1}_{A_{n}}=$ $\lim _{\mathcal{F}}\left(g_{\langle n, k\rangle}\right)_{k}$. Also replacing if necessary $g_{\langle n, k\rangle}$ by $\min \left(1, \max \left(0, g_{\langle n, k\rangle}\right)\right)$ we can assume that each $g_{\langle n, k\rangle}$ is also valued in the unit interval. Then the functions $f_{k}=\sum_{n=0}^{\infty} \alpha_{n} g_{\langle n, k\rangle}$ are well defined and continuous on $X$, and we next check that $f=\lim _{\mathcal{F}} f_{k}$, which will prove that $f \in \mathcal{C}_{\mathcal{F}}(X)$.

So let $x \in X$ and $\varepsilon>0$, and fix $N$ such that $\sum_{n>N} \alpha_{n}<\varepsilon / 3$. Then the set $M=\bigcap_{n=0}^{N}\left\{k:\left|\mathbb{1}_{A_{n}}(x)-g_{\langle n, k\rangle}(x)\right|<\varepsilon /\left(3 N \alpha_{n}\right)\right\}$ is in $\mathcal{F}$ and for all $k \in M$ we have

$$
\left|f(x)-f_{k}(x)\right|<\sum_{n=0}^{N} \alpha_{n}\left|\mathbb{1}_{A_{n}}(x)-g_{\langle n, k\rangle}(x)\right|+2 \sum_{n>N}^{\infty} \alpha_{n}<\varepsilon .
$$

In the general case of an arbitrary function $f \in \mathcal{B}_{\xi}$ pick any homeomorphism $\varphi: \mathbb{R} \rightarrow] 0,1\left[\right.$ and consider the bounded function $g=\varphi \circ f \in \mathcal{B}_{\xi}$. By the previous case $g \in \mathcal{C}_{\mathcal{F}}(X)$; and since $\mathcal{C}_{\mathcal{F}}(X)$ is closed under composition on the left with continuous mappings, it follows that $f=\varphi^{-1} \circ g \in \mathcal{C}_{\mathcal{F}}(X)$.

Lemma 2.2. If a subset $A$ of $X$ is such that $A=\bigcup_{n \in \omega} A_{n}$ and $X \backslash A=$ $\bigcup_{n \in \omega} B_{n}$ with $\mathbb{1}_{A_{n}}, \mathbb{1}_{B_{n}} \in \mathcal{C}_{\mathcal{F}}(X)$ for all $n$, then $\mathbb{1}_{A} \in \mathcal{C}_{\mathcal{F}}(X)$.

Proof. Fix for all $n$ two sequences $\left(f_{\langle n, k\rangle}\right)_{k}$ and $\left(g_{\langle n, k\rangle}\right)_{k}$ of continuous functions such that $\mathbb{1}_{A_{n}}=\lim _{\mathcal{F}}\left(f_{\langle n, k\rangle}\right)_{k}$ and $\mathbb{1}_{B_{n}}=\lim _{\mathcal{F}}\left(g_{\langle n, k\rangle}\right)_{k}$. As in the proof of Lemma 2.1, we may assume that all these functions are valued in $[0,1]$. Then for all $k \in \omega$ the functions $f_{k}=\sum_{n=0}^{\infty} 2^{-n} f_{\langle n, k\rangle}$ and $g_{k}=\sum_{n=0}^{\infty} 2^{-n} g_{\langle n, k\rangle}$ are well defined and continuous; and letting $f=$ $\sum_{n=0}^{\infty} 2^{-n_{1}} A_{A_{n}}$ and $g=\sum_{n=0}^{\infty} 2^{-n_{1}} B_{B_{n}}$ one checks as in the proof of Lemma 2.1 that $f=\lim _{\mathcal{F}} f_{k}$ and $g=\lim _{\mathcal{F}} g_{k}$.

Now since $A=[f>0]$ and $X \backslash A=[g>0]$, we clearly have

$$
\mathbb{1}_{A}=\lim _{\mathcal{F}} \frac{f_{k}}{f_{k}+g_{k}+2^{-k}}
$$

Lemma 2.3. Suppose that $X$ is zero-dimensional. If a subset $A$ of $X$ is such that $\mathbb{1}_{A} \in \mathcal{C}_{\mathcal{F}}(X)$ then there exists a sequence $\left(A_{n}\right)_{n \in \omega}$ of clopen subsets of $X$ such that $\mathbb{1}_{A}=\lim _{\mathcal{F}} \mathbb{1}_{A_{n}}$.

Proof. Let $\left(f_{n}\right)_{n \in \omega}$ be a sequence of continuous functions such that $\mathbb{1}_{A}=$ $\lim _{\mathcal{F}} f_{n}$. Since $X$ is zero-dimensional, we can find for all $n$ a continuous function $g_{n}$ on $X$ with values in $\mathbb{R} \backslash\{1 / 2\}$ and such that $\sup _{x \in X}\left|f_{n}(x)-g_{n}(x)\right|$ $<2^{-n}$; then clearly $\mathbb{1}_{A}=\lim _{\mathcal{F}} g_{n}$. In particular, for all $x \in A$ (resp. $x \notin A$ ) 
the set $\left\{n: g_{n}(x)>1 / 2\right\}$ (resp. $\left.\left\{n: g_{n}(x)<1 / 2\right\}\right)$ is in $\mathcal{F}$. Then for all $n$, $A_{n}=\left[g_{n}>1 / 2\right]$ is a clopen subset of $X$, and it follows from the previous observation that $\mathbb{1}_{A}=\lim _{\mathcal{F}} \mathbb{1}_{A_{n}}$.

We recall that a pointclass $\boldsymbol{\Gamma}$ is said to be a Wadge class if there exists some set $A_{0}$ in $\boldsymbol{\Gamma}\left(\mathbf{2}^{\omega}\right)$ such that for any set $A$ in $\boldsymbol{\Gamma}\left(\mathbf{2}^{\omega}\right)$ there exists a continuous mapping $\varphi: \mathbf{2}^{\omega} \rightarrow \mathbf{2}^{\omega}$ such that $A=\varphi^{-1}\left(A_{0}\right)$.

Lemma 2.4. Suppose that $X$ is zero-dimensional. If $\mathcal{F}$ is $\boldsymbol{\Gamma}$-separated from $\mathcal{F}^{*}$ and $\mathbb{1}_{A} \in \mathcal{C}_{\mathcal{F}}\left(\mathbf{2}^{\omega}\right)$ for all $A \in \boldsymbol{\Gamma}$, then $\boldsymbol{\Gamma}$ is a Wadge class.

Proof. Suppose that $\mathcal{F}$ is separated from $\mathcal{F}^{*}$ by some $\mathcal{G}$ in $\boldsymbol{\Gamma}$. Consider now an arbitrary subset $A$ of $\mathbf{2}^{\omega}$ in $\boldsymbol{\Gamma}$. Since $\mathbb{1}_{A} \in \mathcal{C}_{\mathcal{F}}\left(\mathbf{2}^{\omega}\right)$, Lemma 2.3 yields a sequence $\left(\varphi_{n}\right)$ of characteristic functions of clopen subsets of $\mathbf{2}^{\omega}$ such that $\mathbb{1}_{A}=\lim _{\mathcal{F}} \varphi_{n}$. If $\Phi: \mathbf{2}^{\omega} \rightarrow \mathbf{2}^{\omega}$ denotes the mapping whose $n$th coordinate is $\varphi_{n}$ then $\Phi$ is clearly continuous and

$$
\left\{\begin{array}{l}
x \in A \Rightarrow \Phi(x) \in \mathcal{F} \Rightarrow \Phi(x) \in \mathcal{G} \\
x \notin A \Rightarrow \Phi(x) \in \mathcal{F}^{*} \Rightarrow \Phi(x) \notin \mathcal{G}
\end{array}\right.
$$

hence $A=\Phi^{-1}(\mathcal{G})$. This proves that $\boldsymbol{\Gamma}$ is a Wadge class.

Lemma 2.5. Suppose that $X$ is zero-dimensional and $\mathcal{F}$ is $\boldsymbol{\Sigma}_{1}^{1}$. If $\boldsymbol{\Gamma}$ is a Wadge class and $\mathcal{F}$ is not $\boldsymbol{\Gamma}$-separated from $\mathcal{F}^{*}$ then $\mathbb{1}_{A} \in \mathcal{C}_{\mathcal{F}}(X)$ for any $A \in \boldsymbol{\Gamma}(X)$.

Proof. Fix $A \in \boldsymbol{\Gamma}(X)$. Since $\mathcal{F}$ is not $\boldsymbol{\Gamma}$-separated from $\mathcal{F}^{*}$, by [10, Theorem 3] (see also [11, Corollary 9]) there exists a continuous mapping $\Phi: X \rightarrow \mathbf{2}^{\omega} \approx \mathcal{P}(\omega)$ such that for all $x \in X:$

$$
\left\{\begin{array}{l}
x \notin A \Rightarrow \Phi(x) \in \mathcal{F} \\
x \in A \Rightarrow \Phi(x) \in \mathcal{F}^{*}
\end{array}\right.
$$

Hence if $\varphi_{n}: X \rightarrow\{0,1\}$ denotes the $n$th coordinate of $\Phi: X \rightarrow \mathbf{2}^{\omega}$ then $\left(\varphi_{n}\right)$ is a sequence of continuous functions on $X$ and clearly $\lim _{\mathcal{F}} \varphi_{n}=\mathbb{1}_{B}$ where $B=\mathbf{2}^{\omega} \backslash A$. Hence $\mathbb{1}_{B} \in \mathcal{C}_{\mathcal{F}}(X)$ and consequently $\mathbb{1}_{A} \in \mathcal{C}_{\mathcal{F}}(X)$ too.

TheOREM 2.6. For any $\Sigma_{1}^{1}$ filter $\mathcal{F}$ and any countable ordinal $\xi$, the following conditions are equivalent:

(i) $\mathcal{C}_{\mathcal{F}}\left(\mathbf{2}^{\omega}\right) \subset \mathcal{B}_{\xi}\left(\mathbf{2}^{\omega}\right)$.

(ii) $\mathcal{C}_{\mathcal{F}}(X) \subset \mathcal{B}_{\xi}(X)$ for any zero-dimensional Polish space $X$.

(iii) $\mathcal{C}_{\mathcal{F}}(X) \subset \mathcal{B}_{\xi}(X)$ for any Polish space $X$.

(iv) $\mathcal{F}$ is $\boldsymbol{\Sigma}_{1+\xi^{-}}^{0}$ separated from $\mathcal{F}^{*}$.

Proof. (iii) $\Rightarrow$ (i) is obvious.

(i) $\Rightarrow\left(\right.$ iv): If $\mathcal{F}$ were not $\boldsymbol{\Sigma}_{1+\xi^{-}}^{0}$ separated from $\mathcal{F}^{*}$, consider any $\boldsymbol{\Sigma}_{1+\xi}^{0}$ non- $\boldsymbol{\Delta}_{1+\xi}^{0}$ subset $A$ of $\mathbf{2}^{\omega}$. Then by Lemma 2.5 applied to the Wadge class $\boldsymbol{\Sigma}_{1+\xi}^{0}$ we would have $\mathbb{1}_{A} \in \mathcal{C}_{\mathcal{F}}\left(\mathbf{2}^{\omega}\right)$, which is impossible since $A \notin \mathbf{\Pi}_{1+\xi}^{0}$. 
(iv) $\Rightarrow$ (ii): Fix a $\boldsymbol{\Sigma}_{1+\xi}^{0}$ set $\mathcal{G}$ containing $\mathcal{F}$ and disjoint from $\mathcal{F}^{*}$, and let $X$ be a zero-dimensional Polish space. Consider a function $f \in \mathcal{C}_{\mathcal{F}}(X)$ and fix some sequence of continuous functions $f_{n}: X \rightarrow \mathbb{R}$ such that $f=\lim _{\mathcal{F}} f_{n}$. Let $a<b$; we have to prove that $f^{-1}(] a, b[) \in \Sigma_{1+\xi}^{0}(X)$.

Let $Q_{(a, b)}$ denote the set of all pairs $(r, s)$ of rational numbers $r, s$ such that $a<r<s<b$. For any $(r, s) \in Q_{(a, b)}$ pick some $\left(r^{\prime}, s^{\prime}\right)$ such that $a<r^{\prime}<r<s<s^{\prime}<b$. Then for any $n \in \omega, f_{n}^{-1}([r, s])$ is a closed set which is contained in the open set $f_{n}^{-1}(] r^{\prime}, s^{\prime}[)$, and since $X$ is zero-dimensional, we can find a clopen set $A_{n}^{(r, s)}$ such that

$$
f_{n}^{-1}([r, s]) \subset A_{n}^{(r, s)} \subset f_{n}^{-1}(] r^{\prime}, s^{\prime}[) .
$$

Let $\Phi_{(r, s)}: X \rightarrow \mathbf{2}^{\omega}$ denote the mapping whose $n$th coordinate is the characteristic function of $A_{n}^{(r, s)}$. Since each $\Phi_{(r, s)}$ is continuous, (ii) follows from the equality

$$
f^{-1}(] a, b[)=\bigcup_{(r, s) \in Q_{(a, b)}} \Phi_{(r, s)}^{-1}(\mathcal{G}),
$$

which we shall prove by checking the two corresponding inclusions.

If $x \in f^{-1}(] a, b[)$ then we can find $(r, s) \in Q_{(a, b)}$ such that $x \in f^{-1}(] r, s[)$, and since $f=\lim _{\mathcal{F}} f_{n}$, the set $\left\{n: x \in f_{n}^{-1}(] r, s[)\right\}$ is in $\mathcal{F}$; a fortiori the larger set $\left\{n: x \in A_{n}^{(r, s)}\right\}$ is in $\mathcal{F}$, hence in $\mathcal{G}$, which proves that $x \in \Phi_{(r, s)}^{-1}(\mathcal{G})$.

Conversely, suppose that $x \in \Phi_{(r, s)}^{-1}(\mathcal{G})$ for some $(r, s) \in Q_{(a, b)}$ and pick $\left(r^{\prime}, s^{\prime}\right) \in Q_{(a, b)}$ such that $\left.f\left(A_{n}^{(r, s)}\right) \subset\right] r^{\prime}, s^{\prime}[$ for all $n$. Then the set $\left\{n: x \in A_{n}^{(r, s)}\right\}$, which is in $\mathcal{G}$, is not in $\mathcal{F}^{*}$, and a fortiori the larger set $\left\{n: x \in f_{n}^{-1}(] r^{\prime}, s^{\prime}[)\right\}$ is not in $\mathcal{F}^{*}$ either. This proves that $x \in f^{-1}\left(\left[r^{\prime}, s^{\prime}\right]\right)$, for otherwise $\left\{n: x \notin f_{n}^{-1}\left(\left[r^{\prime}, s^{\prime}\right]\right)\right\}$ would be in $\mathcal{F}$, or equivalently $\{n$ : $\left.x \in f_{n}^{-1}\left(\left[r^{\prime}, s^{\prime}\right]\right)\right\}$ would be in $\mathcal{F}^{*}$, which contradicts the previous conclusion. Hence $x \in f^{-1}\left(\left[r^{\prime}, s^{\prime}\right]\right) \subset f^{-1}(] a, b[)$, which finishes the proof of the equality above.

(ii) $\Rightarrow$ (iii): Let $X$ be an arbitrary Polish space and fix any continuous surjection $\hat{\pi}: \mathbf{2}^{\omega} \rightarrow \hat{X}$ from $\mathbf{2}^{\omega}$ onto some compactification $\hat{X}$ of $X$. Then $Y=\hat{\pi}^{-1}(X)$ is a zero-dimensional Polish space and the mapping $\pi: Y \rightarrow X$ obtained by restriction of $\hat{\pi}$ is perfect.

Consider now any $f \in \mathcal{C}_{\mathcal{F}}(X)$ and fix a sequence of continuous functions $f_{n}: X \rightarrow \mathbb{R}$ such that $f=\lim _{\mathcal{F}} f_{n}$. Then the functions $g_{n}=\pi \circ f_{n}$ are continuous on $Y$, and by the continuity of $\pi, g:=\pi \circ f=\lim _{\mathcal{F}} g_{n}$, which proves that $g \in \mathcal{C}_{\mathcal{F}}(Y)$, hence by (ii), $g=\pi \circ f \in \mathcal{B}_{\xi}$. It then follows from [14] that $f \in \mathcal{B}_{\xi}$.

REMARK 2.7. At first glance condition (ii) of Theorem 2.6 might appear insignificant, trivially sitting between (i) and (iii). However, its role in the above proof is crucial. Indeed, because of the compactness of $\mathbf{2}^{\omega}$ there is no 
direct way of deriving (iii) from (i) and the only proof we can produce for the (most significant) implication (iv) $\Rightarrow$ (iii) is via (ii).

TheOREM 2.8. For any $\boldsymbol{\Sigma}_{1}^{1}$ filter $\mathcal{F}$ and any countable ordinal $\xi$, the following conditions are equivalent:

(i) $\mathcal{B}_{\xi}(X) \subset \mathcal{C}_{\mathcal{F}}(X)$ for any zero-dimensional Polish space $X$.

(ii) For all $A \in \bigcup_{\eta<\xi} \boldsymbol{\Sigma}_{1+\eta}^{0}\left(\mathbf{2}^{\omega}\right), \mathbb{1}_{A} \in \mathcal{C}_{\mathcal{F}}\left(\mathbf{2}^{\omega}\right)$.

(iii) For all $\eta<\xi, \mathcal{F}$ is not $\Sigma_{1+\eta}^{0}$-separated from $\mathcal{F}^{*}$.

(iv) $\xi=0$ or $\mathcal{F}$ is not $\boldsymbol{\Delta}_{1+\xi}^{0}$-separated from $\mathcal{F}^{*}$.

Proof. Since for $\xi=0$ all four conditions are trivially true, we may suppose that $\xi>0$. Observe first that the implications (i) $\Rightarrow$ (ii) and (iv) $\Rightarrow$ (iii) are obvious.

(ii) $\Rightarrow$ (iv): It follows from (ii) and Lemma 2.2 that $\mathbb{1}_{A} \in \mathcal{C}_{\mathcal{F}}\left(\mathbf{2}^{\omega}\right)$ for all $A \in \boldsymbol{\Delta}_{1+\xi}^{0}$. Hence by Lemma 2.4 , if $\mathcal{F}$ were $\boldsymbol{\Delta}_{1+\xi^{-}}^{0}$-separated from $\mathcal{F}^{*}$ then $\boldsymbol{\Delta}_{1+\xi}^{0}$ would be a Wadge class, which is not the case since $\xi>0$.

(iii) $\Rightarrow$ (i): By Lemmas 2.1 and Lemma 2.2, to prove (i) it is enough to show that $\mathbb{1}_{A} \in \mathcal{C}_{\mathcal{F}}(X)$ for all $A \in \bigcup_{\eta<\xi} \Sigma_{1+\eta}^{0}(X)$; and this follows from Lemma 2.5 applied to the Wadge classes $\boldsymbol{\Sigma}_{1+\eta}^{0}$.

REMARK 2.9. It will follow from the main result of Section 7 that if $\xi \leq 2$ then the zero-dimensionality condition on $X$ in (i) of Theorem 2.8 can be dropped. We conjecture that this is the case for any ordinal $\xi$. As we shall see, this seemingly soft topological problem is related to other deep descriptive problems (see Conjecture 7.8).

The following result answers a question asked by Solecki in [15] (see the Remark preceding Theorem 1.4 there, where the particular case $\xi=3$ is proved). Recall that a filter $\mathcal{F}$ is said to be free if $\bigcap \mathcal{F}=\emptyset$.

Corollary 2.10. Any $\Pi_{\xi}^{0}$ free filter $\mathcal{F}$ is separated from its ideal $\mathcal{F}^{*}$ by a $\Sigma_{\eta}^{0}$ set for some $\eta<\xi$.

Proof. Observe first that since $\mathcal{F}$ is free, necessarily $\xi \geq 3$. Now since $\mathcal{F}$ is $\boldsymbol{\Pi}_{\xi}^{0}$, so is its ideal $\mathcal{F}^{*}$; it then follows from Lemma 1.4 that $\mathcal{F}$ is $\boldsymbol{\Delta}_{\xi}^{0}$-separated from $\mathcal{F}^{*}$, and since $\xi \geq 3$, Theorem 2.8 implies that $\mathcal{F}$ is $\boldsymbol{\Sigma}_{\eta}^{0}$-separated from $\mathcal{F}^{*}$ for some $\eta<\xi$.

REMARK 2.11. It follows from Lemma 1.3 of [15] and Corollary 2.10 that if $\mathcal{F}$ is a $\boldsymbol{\Pi}_{\xi}^{0}$ filter then the set

$$
\operatorname{Conv}(\mathcal{F}):=\left\{\left(x_{n}\right)_{n \in \omega} \in \mathbb{R}^{\omega}:\left(x_{n}\right) \text { converges relative to } \mathcal{F}\right\}
$$

is itself a $\Pi_{\xi}^{0}$ subset of $\mathbb{R}^{\omega}$, which solves a problem raised by Dobrowolski and Marciszewski in [3]. 


\section{The Borel separation rank of a filter}

Definition 3.1. The Borel separation rank (or more simply the rank) of a filter $\mathcal{F}$ is the unique ordinal defined by

$$
\operatorname{rk}(\mathcal{F})=\min \left\{\xi<\omega_{1}: \mathcal{F} \text { is } \boldsymbol{\Sigma}_{1+\xi^{-}}^{0} \text { separated from } \mathcal{F}^{*}\right\} .
$$

So $\operatorname{rk}(\mathcal{F})<\omega_{1}$ if and only if $\mathcal{F}$ is $\boldsymbol{\Delta}_{1}^{1}$-separated from $\mathcal{F}^{*}$. In particular, by the Suslin separation theorem, any $\boldsymbol{\Sigma}_{1}^{1}$ filter admits a countable rank.

It follows readily from the definition above that any of the four conditions in Theorem 2.6 is equivalent to saying that $\operatorname{rk}(\mathcal{F}) \leq \xi$. Similarly, any of the four conditions in Theorem 2.8 is equivalent to $\operatorname{rk}(\mathcal{F}) \geq \xi$, and combining these two results one gets:

TheOREM 3.2. For any $\boldsymbol{\Sigma}_{1}^{1}$ filter $\mathcal{F}$ and any countable ordinal $\xi>0$, the following conditions are equivalent:

(i) $\operatorname{rk}(\mathcal{F})=\xi$.

(ii) $\mathcal{C}_{\mathcal{F}}(X)=\mathcal{B}_{\xi}(X)$ for any zero-dimensional Polish space $X$.

(iii) $\mathcal{F}$ is $\boldsymbol{\Sigma}_{1+\xi^{-}}^{0}$ eparated but not $\boldsymbol{\Delta}_{1+\xi^{-}}^{0}$ separated from $\mathcal{F}^{*}$.

(iv) $\xi=\min \left\{\eta: \mathcal{F}\right.$ is $\boldsymbol{\Sigma}_{1+\eta^{-}}^{0}$ separated from $\left.\mathcal{F}^{*}\right\}$.

(v) $\xi=\max \left\{\eta: \mathcal{F}\right.$ is not $\boldsymbol{\Delta}_{1+\eta^{-}}^{0}$ separated from $\left.\mathcal{F}^{*}\right\}$.

For $\xi=0$ we have the following simple characterization which does not necessitate any descriptive regularity on the filter.

Proposition 3.3. A filter $\mathcal{F}$ is of rank 0 if and only if $\mathcal{F}$ is not free.

Proof. If $\mathcal{F}$ is free then for all $m \in \omega$ the singleton $\{m\}$ belongs to $\mathcal{F}^{*}$ and so does the finite set $J_{m}=\{p: p \leq m\}$. But since in $\mathcal{P}(\omega)$ we have $\lim _{m \rightarrow \infty} J_{m}=\omega \in \mathcal{F}$, we conclude that $\mathcal{F}$ meets the closure of $\mathcal{F}^{*}$, and $\mathcal{F}$ is not $\boldsymbol{\Sigma}_{1}^{0}$-separated from $\mathcal{F}^{*}$, hence $\operatorname{rk}(\mathcal{F})>0$.

Conversely, if $\mathcal{F}$ is not $\boldsymbol{\Sigma}_{1}^{0}$-separated from $\mathcal{F}^{*}$ then there exists some $M \in \mathcal{F}$ which is in the closure of $\mathcal{F}^{*}$, and we can find a sequence $\left(M_{n}\right)$ in $\mathcal{F}^{*}$ which converges to $M$. Thus defining $M_{n}^{\prime}$ by $M_{n}^{\prime}=(\omega \backslash M) \cup M_{n}$, we get a sequence of elements of $\mathcal{F}^{*}$ which converges to $\omega$ in $\mathcal{P}(\omega) \approx 2^{\omega}$. For every $n$ choose an integer $k_{n} \notin \bigcup_{p \leq n} M_{p}^{\prime}$ and define the continuous function $\varphi_{n}: \mathcal{P}(\omega) \rightarrow\{0,1\}$ by $\varphi_{n}(M)=1 \Leftrightarrow \forall p \leq n, k_{p} \in M$. Then the sequence $\left(\varphi_{n}\right)$ is non-increasing and converges to some function $\varphi$ along the Fréchet filter $\mathcal{N}$, hence along $\mathcal{F}$ since $\mathcal{N} \subset \mathcal{F}$. Clearly, $\varphi(\omega)=1$ and $\varphi_{k}\left(M_{n}^{\prime}\right)=0$ for all $k>n$, hence $\varphi\left(M_{n}^{\prime}\right)=0$ for all $n$, which shows that $\varphi$ is not continuous. This proves that $\mathcal{F}$ is free: if there were some integer $k$ in $\bigcap \mathcal{F}$ then necessarily $\varphi=\varphi_{k}$ and $\varphi$ would be continuous.

In the two coming sections we shall study the behaviour of the rank function relative to some natural operations on filters. Let us only point out here the following simple property: 
Proposition 3.4. Given two filters $\mathcal{F}$ and $\mathcal{G}$, if $\mathcal{F} \subset \mathcal{G}$ then $\operatorname{rk}(\mathcal{F}) \leq$ $\operatorname{rk}(\mathcal{G})$.

Proof. Observe that if $\mathcal{F} \subset \mathcal{G}$ then $\mathcal{F}^{*} \subset \mathcal{G}^{*}$, and any set separating $\mathcal{G}$ from $\mathcal{G}^{*}$ also separates $\mathcal{F}$ from $\mathcal{F}^{*}$.

4. Sum operations on filters. The operations considered in this section are quite standard. Since there is no universal terminology in this area we shall fix one for our own use.

For any family $\left(E_{i}\right)_{i \in I}$ of sets we denote by $\sum_{i \in I} E_{i}$ its disjoint union.

4.1. Fubini sums of filters. If $\left(\mathcal{F}_{i}\right)_{i \in I}$ is a family of filters and $\mathcal{A}$ is a filter on $I$ then the set of all sets of the form

$$
\left\{\sum_{i \in J} M_{i}: \forall i \in J, M_{i} \in \mathcal{F}_{i}\right\}
$$

for $J \in \mathcal{A}$ is the basis of a filter $\mathcal{F}$ on $\sum_{i \in I} \operatorname{dom}\left(\mathcal{F}_{i}\right)$ that we call the $\mathcal{A}$-Fubini sum of the family $\left(\mathcal{F}_{i}\right)_{i \in I}$.

Of most importance for what follows is the case where $I$ is a directed set and $\mathcal{A}$ is the canonical filter generated by the sets of the form $J_{i}=\{j \in I$ : $j \geq i$ \}. In this case we shall say more simply that $\mathcal{F}$ is the Fubini sum of the family $\left(\mathcal{F}_{i}\right)_{i \in I}$.

We now point out two particular instances of the Fubini sum operation.

4.2. Complete sums of filters. Observe that in the particular case where $\mathcal{A}=\{I\}$ is the trivial filter on $I$, the set of all sets of the form

$$
\left\{\sum_{i \in I} M_{i}: \forall i \in I, M_{i} \in \mathcal{F}_{i}\right\}
$$

is already a filter on $\sum_{i \in I} \operatorname{dom}\left(\mathcal{F}_{i}\right)$ that we call the complete sum (or simply the sum) filter of the family $\left(\mathcal{F}_{i}\right)_{i \in I}$.

4.3. Extensions of filters. Given a filter $\mathcal{F}$ we shall say that a filter $\mathcal{G}$ is an $\mathcal{A}$-extension of $\mathcal{F}$ if $\mathcal{G}$ is the $\mathcal{A}$-Fubini sum of the family $\left(\mathcal{F}_{i}\right)_{i \in I}$ with $\mathcal{F}_{i}=\mathcal{F}$ for all $i$. If moreover $\mathcal{A}$ is the Fréchet filter then we shall say that $\mathcal{G}$ is a Fréchet extension of $\mathcal{F}$.

Proposition 4.4. Suppose that $\mathcal{G}$ is the $\mathcal{A}$-Fubini sum of the family $\left(\mathcal{F}_{i}\right)_{i \in I}$ and let $J \subset I$ be an element of $\mathcal{A}$.

(a) If $\operatorname{rk}(\mathcal{A}) \geq \alpha$ and $\operatorname{rk}\left(\mathcal{F}_{i}\right) \geq \xi$ for all $i \in J$ then $\operatorname{rk}(\mathcal{G}) \geq \xi+\alpha$.

(b) If $\operatorname{rk}(\mathcal{A}) \leq \alpha$ and $\operatorname{rk}\left(\mathcal{F}_{i}\right) \leq \xi$ for all $i \in J$ then $\operatorname{rk}(\mathcal{G}) \leq \xi+1+\alpha$.

(c) If $\mathcal{A}$ is the Fréchet filter and $\operatorname{rk}\left(\mathcal{F}_{i}\right) \leq \xi$ for all $i \in J$ then $\operatorname{rk}(\mathcal{G}) \leq$ $\xi+1$.

Proof. Let $D_{i}=\operatorname{dom}\left(\mathcal{F}_{i}\right)$ and $D=\sum_{i \in I} D_{i}$ be the domain of $\mathcal{G}$. 
(a) Let $\tau_{0}$ denote the standard topology on $\mathbf{2}^{\omega}$. It is well known that given any function $g \in \mathcal{B}_{\xi+\alpha}\left(\mathbf{2}^{\omega}\right)$ one can find a zero-dimensional Polish topology $\tau$ on $\mathbf{2}^{\omega}$ with $\boldsymbol{\Sigma}_{1}^{0}\left(\tau_{0}\right) \subset \boldsymbol{\Sigma}_{1}^{0}(\tau) \subset \boldsymbol{\Sigma}_{1+\xi}^{0}\left(\tau_{0}\right)$ and such that $g$ is of class $\alpha$ relative to $\tau$. So for $X=\left(\mathbf{2}^{\omega}, \tau\right)$ the identity mapping $h: \mathbf{2}^{\omega} \rightarrow X$ is of class $\xi$, and $g=f \circ h$ with some $f: X \rightarrow \mathbb{R}$ of class $\alpha$. Since $\operatorname{rk}(\mathcal{A}) \geq \alpha$, there exists a family $\left(f_{i}\right)_{i \in I}$ of continuous functions on $X$ converging to $f$ along $\mathcal{A}$. Then for each $i \in J$ the function $f_{i} \circ h$ is of class $\xi$ on $\mathbf{2}^{\omega}$, hence is the limit along $\mathcal{F}_{i}$ of a sequence of continuous functions $f_{i, n}: 2^{\omega} \rightarrow \mathbb{R}$. Then setting $f_{i, n}=0$ for $n \in \omega$ and $i \in I \backslash J$ one easily checks that the family $\left(f_{i, n}\right)_{(i, n) \in I \times \omega}$ converges to $g$ along $\mathcal{G}$.

(b) For all $i \in J, \operatorname{since} \operatorname{rk}\left(\mathcal{F}_{i}\right) \leq \xi$ we can pick a $\boldsymbol{\Sigma}_{1+\xi}^{0}$ set $S_{i} \subset \mathcal{P}\left(D_{i}\right)$ separating $\mathcal{F}_{i}$ from $\mathcal{F}_{i}^{*}$. Then each mapping $\psi_{i}: \mathcal{P}(D) \rightarrow\{0,1\}$ defined by

$$
\psi_{i}(M)= \begin{cases}1 & \text { if } i \in J \text { and } M \cap D_{i} \in S_{i}, \\ 0 & \text { if not, }\end{cases}
$$

is of class $\xi+1$, and so is the mapping $\psi: \mathcal{P}(D) \rightarrow\{0,1\}^{I} \approx \mathcal{P}(I)$ defined by $\psi(M)=\left(\psi_{i}(M)\right)_{i \in I}$.

Since $\operatorname{rk}(\mathcal{A}) \leq \alpha$ we can also fix a $\boldsymbol{\Sigma}_{1+\alpha}^{0}$ set $S \subset \mathcal{P}(I)$ separating $\mathcal{A}$ from $\mathcal{A}^{*}$. Then $\tilde{S}=\psi^{-1}(S) \in \Sigma_{1+\xi+1+\alpha}^{0}$ and one readily checks that $\tilde{S}$ separates $\mathcal{G}$ from $\mathcal{G}^{*}$. Hence $\operatorname{rk}(\mathcal{G}) \leq \xi+1+\alpha$.

(c) If $\mathcal{A}=\mathcal{N}$ is the Fréchet filter then $S:=\{M \subset \omega: \forall i \exists j \geq i, j \in M\}$ is a $\Pi_{2}^{0}$ set separating $\mathcal{N}$ from $\mathcal{N}^{*}$ and as above $\tilde{S}:=\psi^{-1}(S)$ separates $\mathcal{G}$ from $\mathcal{G}^{*}$. But since $\tilde{S}=\bigcap_{i} \bigcup_{j \geq i}\left\{M \in \mathcal{P}(D): M \cap D_{i} \in S_{i}\right\}$, it is clearly a $\Pi_{1+\xi+1}^{0}$ set, which shows that $\operatorname{rk}(\mathcal{G}) \leq \xi+1$.

Proposition 4.5. If $\mathcal{G}$ is the complete sum filter of the family $\left(\mathcal{F}_{i}\right)_{i \in I}$ then $\operatorname{rk}(\mathcal{G})=\min _{i \in I} \operatorname{rk}\left(\mathcal{F}_{i}\right)$.

Proof. One easily checks that a function $f: X \rightarrow \mathbb{R}$ is the limit along $\mathcal{G}$ of a family $\left(f_{i, n}\right)_{i \in I, n \in \operatorname{dom}\left(\mathcal{F}_{i}\right)}$ of functions if and only if, for all $i \in I, f$ is the limit along $\mathcal{F}_{i}$ of $\left(f_{i, n}\right)_{n \in \operatorname{dom}\left(\mathcal{F}_{i}\right)}$. And for a function $f: X \rightarrow \mathbb{R}$ of Borel class $\xi$ on a zero-dimensional space $X$ this is possible if and only if $\xi \leq \operatorname{rk}\left(\mathcal{F}_{i}\right)$ for all $i \in I$.

Proposition 4.6. Let $\xi$ be an ordinal, $\mathcal{A}$ be a filter with countable basis, and let $\mathcal{G}$ be the $\mathcal{A}$-Fubini sum of the family $\left(\mathcal{F}_{i}\right)_{i \in I}$. If for all $i \in I, \mathcal{F}_{i}$ is a $\boldsymbol{\Sigma}_{\eta}^{0}$ subset of $\mathcal{P}\left(\operatorname{dom}(\mathcal{F})_{i}\right)$ for some $\eta<\xi$ then $\mathcal{G}$ is a $\boldsymbol{\Sigma}_{\xi+1}^{0}$ set.

Proof. Denote by $\left(A_{k}\right)$ a basis of $\mathcal{A}$, by $D_{i}$ the domain of $\mathcal{F}_{i}$, and by $D=\sum D_{i}$ the domain of $\mathcal{G}$. Then $M \in \mathcal{G}$ if and only if there is some $A \in \mathcal{A}$ such that $M \cap D_{i} \in \mathcal{F}_{i}$ for all $i \in A$, hence

$$
\mathcal{G}=\bigcup_{k} \bigcap_{i \in A_{k}}\left\{M \in \mathcal{P}(D): M \cap D_{i} \in \mathcal{F}_{i}\right\}
$$


And since the mapping $M \mapsto M \cap D_{i}$ is continuous, each set $\{M \in \mathcal{P}(D)$ : $\left.M \cap D_{i} \in \mathcal{F}_{i}\right\}$ is $\Pi_{\xi}^{0}$.

Notice that in the particular case of complete sums we have the following stronger conclusion obtained by a straightforward complexity computation.

Proposition 4.7. Let $\mathcal{G}$ be the complete sum filter of the family $\left(\mathcal{F}_{i}\right)_{i \in I}$. If , for all $i \in I, \mathcal{F}_{i}$ is a $\Pi_{\xi}^{0}$ subset of $\mathcal{P}\left(\operatorname{dom}(\mathcal{F})_{i}\right)$ then $\mathcal{G}$ is a $\Pi_{\xi}^{0}$ set.

5. Inductive limits. In this section we shall define a kind of "union" operation on families of filters with variable domains. For this we need the following slight modification of the notion of morphism or homomorphism between filters introduced by Katětov (see [4] or [5]).

Definition 5.1. A quasi-homomorphism from the filter $\mathcal{F}$ to the filter $\mathcal{G}$ is a mapping $\pi: F \rightarrow \operatorname{dom}(\mathcal{G})$ with domain $F \in \mathcal{F}$ and such that for all $M \in \mathcal{G}, \pi^{-1}(M) \in \mathcal{F}$.

LEMMA 5.2. If there exists a quasi-homomorphism from the filter $\mathcal{F}$ to the filter $\mathcal{G}$ then $\operatorname{rk}(\mathcal{G}) \leq \operatorname{rk}(\mathcal{F})$.

Proof. Let $\xi$ be the rank of $\mathcal{F}, \pi: F \rightarrow \operatorname{dom}(\mathcal{G})$ be a quasi-homomorphism and $S \subset \mathcal{P}(\operatorname{dom}(\mathcal{F}))$ be a $\Sigma_{1+\xi}^{0}$ set separating $\mathcal{F}$ from $\mathcal{F}^{*}$. Then the set

$$
S^{\prime}:=\left\{M \in \mathcal{P}(\operatorname{dom}(\mathcal{G})): \pi^{-1}(M) \in S\right\}
$$

is $\boldsymbol{\Sigma}_{1+\xi}^{0}$. If $M \in \mathcal{G}$ then $\pi^{-1}(M) \in \mathcal{F} \subset S$.

If $M \in \mathcal{G}^{*}$ then the complement $N:=\operatorname{dom}(\mathcal{G}) \backslash M$ belongs to $\mathcal{G}$. Hence $\pi^{-1}(N) \in \mathcal{F}$ and $\pi^{-1}(M)=F \backslash \pi^{-1}(N) \in \mathcal{F}^{*} \subset \mathcal{P}(\operatorname{dom}(\mathcal{F})) \backslash S$. Thus $S^{\prime}$ separates $\mathcal{G}$ from $\mathcal{G}^{*}$, and $\operatorname{rk}(\mathcal{G}) \leq \xi$.

Definition 5.3. Let $(I, \leq)$ be a directed set and $\left(\mathcal{F}_{i}\right)_{i \in I}$ be a family of filters. We shall say that the family $\left(\pi_{i, j}\right)_{i \leq j}$ is a coherent system of quasihomomorphisms for $\left(\mathcal{F}_{i}\right)_{i \in I}$ if for all $i \leq j \leq k$ in $I$ :

- $\pi_{i, j}$ is a quasi-homomorphism from $\mathcal{F}_{j}$ to $\mathcal{F}_{i}$.

- For any $a \in \operatorname{dom}\left(\pi_{i, k}\right) \cap \operatorname{dom}\left(\pi_{j, k}\right) \cap \pi_{j, k}^{-1}\left(\operatorname{dom}\left(\pi_{i, j}\right)\right)$ we have

$$
\pi_{i, k}(a)=\pi_{i, j}\left(\pi_{j, k}(a)\right) .
$$

We shall then say that $\left(\mathcal{F}_{i}, \pi_{i, j}\right)_{i \leq j}$ is a quasi-inductive system of filters.

Proposition-Definition 5.4. Let $\left(\pi_{i, j}\right)_{i \leq j}$ be a coherent system of quasi-homomorphisms for the family $\left(\mathcal{F}_{i}\right)_{i \in I}$ of filters. Then the set of all sets of the form $\bigcup_{j \geq i} \pi_{i, j}^{-1}(M)$ for some $i$ and $M \in \mathcal{F}_{i}$ constitutes a basis of a filter on $\sum_{i \in I} \operatorname{dom}\left(\mathcal{F}_{i}\right)$.

The filter generated by this basis will be denoted by $\lim _{\mathcal{F}} \mathcal{F}_{i}$ (when there is no ambiguity on the $\pi_{i, j}$ ) and called the inductive limit of the system $\left(\mathcal{F}_{i}, \pi_{i, j}\right)_{i \leq j}$. 
Moreover, for every $i \in I$ there is a quasi-homomorphism $\pi_{i}$ from $\lim \mathcal{F}_{i}$ to $\mathcal{F}_{i}$ such that for all $i \leq j, \pi_{i}$ and $\pi_{i, j} \circ \pi_{j}$ agree on $\operatorname{dom}\left(\pi_{j}\right) \cap \pi_{j}^{-1}\left(\operatorname{dom}\left(\pi_{i, j}\right)\right)$.

Proof. For $i \in I$ and $M \in \mathcal{F}_{i}$ define $\hat{M}=\bigcup_{j \geq i} \pi_{i, j}^{-1}(M)$. We first prove that the family $\left\{\hat{M}: i \in I, M \in \mathcal{F}_{i}\right\}$ is a basis of a filter. Indeed, let $i, j \in I$, $M \in \mathcal{F}_{i}, N \in \mathcal{F}_{j}$. Choose $k \in I$ such that $i, j \leq k$ and define

$$
P:=\pi_{i, k}^{-1}(M) \cap \pi_{j, k}^{-1}(N) \in \mathcal{F}_{k} .
$$

We show that $\hat{P} \subset \hat{M} \cap \hat{N}$. So let $x \in \hat{P}$. There exists $l \geq k$ such that $x \in \operatorname{dom}\left(\pi_{k, l}\right) \subset \operatorname{dom}\left(\mathcal{F}_{l}\right)$ and $y:=\pi_{k, l}(x) \in P$. By coherence we conclude that $\pi_{i, k}(y) \in M$ and $\pi_{j, k}(y) \in N$, hence $x \in \hat{M} \cap \hat{N}$.

Let $i \in I$ and define $F_{i}:=\bigcup_{j \geq i} \operatorname{dom}\left(\pi_{i, j}\right) \subset \sum_{j \in I} \operatorname{dom}\left(\mathcal{F}_{j}\right)$.

Since $M_{0}=\operatorname{dom}\left(\mathcal{F}_{i}\right) \in \mathcal{F}_{i}$, we have $F_{i}=\hat{M}_{0}$, hence $F_{i} \in \mathcal{F}:=\lim _{\longleftarrow} \mathcal{F}_{j}$. Define $\pi_{i}: F_{i} \rightarrow \operatorname{dom}\left(\mathcal{F}_{i}\right)$ by

$$
\pi_{i}(a)=\pi_{i, j}(a) \quad \text { if } j \geq i \text { and } a \in \operatorname{dom}\left(\pi_{i, j}\right) \subset \operatorname{dom}\left(\mathcal{F}_{j}\right) .
$$

It is then easily checked that $\pi_{i}$ is a quasi-homomorphism from $\mathcal{F}$ to $\mathcal{F}_{i}$. Moreover, if $i \leq j$ and $a \in F_{j} \subset F_{i}$ there is a $k \geq j \geq i$ such that $a \in \operatorname{dom}\left(\pi_{j, k}\right)$ and $\pi_{j}(a)=\pi_{j, k}(a)$. Then the conclusion follows from the coherence condition.

Proposition 5.5. Let $\mathcal{F}$ be the limit of the quasi-inductive system $\left(\mathcal{F}_{i}, \pi_{j, i}\right)_{i \leq j}$ of filters.

(a) If $\operatorname{rk}\left(\mathcal{F}_{i}\right) \geq \xi$ for some $i$ then $\operatorname{rk}(\mathcal{F}) \geq \xi$.

(b) If $\operatorname{rk}\left(\mathcal{F}_{i}\right) \leq \xi$ for all $i$ then $\operatorname{rk}(\mathcal{F}) \leq \xi+1$.

Proof. Statement (a) immediately follows from Lemma 5.2 and Proposition 5.4.

Denote by $D_{i}$ the domain of $\mathcal{F}_{i}$ and by $D=\sum_{i \in I} D_{i}$ the domain of $\mathcal{F}$. Choose for each $i \in I$ a $\Pi_{1+\xi}^{0}$ set $S_{i}$ separating $\mathcal{F}_{1}$ from $\mathcal{F}_{i}^{*}$ in $\mathcal{P}\left(D_{i}\right)$, and define

$$
S:=\left\{M \in \mathcal{P}(D): \exists i \forall j \geq i, M \cap D_{j} \in S_{j}\right\} .
$$

Since the mapping $M \mapsto M \cap D_{i}$ is continuous from $\mathcal{P}(D)$ to $\mathcal{P}\left(D_{i}\right)$ for every $i$, it is clear that $S$ is $\boldsymbol{\Sigma}_{1+\xi+1}^{0}$ in $\mathcal{P}(D)$.

If $M \in \mathcal{F}$ there are $i \in I$ and $N \subset D_{i}$ in $\mathcal{F}_{i}$ such that $\hat{N} \subset M$. Then for $j \geq i$ we have $M \cap D_{j} \supset \hat{N} \cap D_{j}=\pi_{i, j}^{-1}(N) \in \mathcal{F}_{j}$, hence $M \cap D_{j} \in \mathcal{F}_{j} \subset S_{j}$. This shows that $M \in S$.

Conversely, if $M \in \mathcal{F}^{*}$ there are $k \in I$ and $N \subset D_{k}$ in $\mathcal{F}_{k}$ such that $M$ and $\hat{N}$ are disjoint. Then for every $i$ in $I$ there is a $j \in I$ such that $j \geq i$ and $j \geq k$, and since $M \cap D_{j}$ is disjoint from $\hat{N} \cap D_{j}=\pi_{k, j}^{-1}(N) \in \mathcal{F}_{j}$ we conclude that $M \cap D_{j} \in \mathcal{F}_{j}^{*} \subset \mathcal{P}\left(D_{j}\right) \backslash S_{j}$, hence $M \notin S$.

So $S$ separates $\mathcal{F}$ from $\mathcal{F}^{*}$, and $\operatorname{rk}(\mathcal{F}) \leq \xi+1$. 
REMARK 5.6. The limit of a quasi-inductive system of filters of rank $\xi$ might be of rank $\xi+1$. To see this consider for all $i \in I=\omega$ the filter

$$
\mathcal{F}_{i}=\{M \subset \omega \times \omega: \forall p \geq i,\{q:(p, q) \in M\} \text { is cofinite }\}
$$

with domain $D_{i}=\omega \times \omega$ and the identity mappings $\pi_{i, j}(p, q)=(p, q)$ for all $i \leq j$ as a system of quasi-homomorphisms, which is clearly coherent.

Then each $\mathcal{F}_{i}$ is of rank 1: indeed, a family $\left(f_{p, q}\right)$ of continuous functions converges to a function $f$ along $\mathcal{F}_{i}$ only if $f=\lim _{q} f_{i, q}$, hence $f$ is of Baire class 1.

To see that $\mathcal{F}$ is of rank $\geq 2$ consider the mapping $\psi:(p, q, i) \mapsto(p, q)$ from $\sum_{i \in \omega} D_{i} \approx \omega \times \omega \times \omega$ to $\omega \times \omega$ : one easily checks that $\psi$ is a quasihomomorphism from $\mathcal{N}_{2}$ to $\mathcal{F}:=\lim \mathcal{F}_{i}$, where $\mathcal{N}_{2}$ is the Fréchet extension of the Fréchet filter, hence by Lemma 5.2 and Proposition $4.4(\mathrm{a}), \operatorname{rk}(\mathcal{F}) \geq$ $\operatorname{rk}\left(\mathcal{N}_{2}\right) \geq 2$.

Proposition 5.7. Let $\mathcal{F}$ be the limit of the quasi-inductive system $\left(\mathcal{F}_{i}, \pi_{j, i}\right)_{i \leq j}$ of filters. If each filter $\mathcal{F}_{i}$ is $\boldsymbol{\Sigma}_{\eta_{i}}^{0}$ in $\mathcal{P}\left(\operatorname{dom}\left(\mathcal{F}_{i}\right)\right)$ for some $\eta_{i}<\xi$ then $\mathcal{F}$ is $\boldsymbol{\Sigma}_{\xi}^{0}$.

Proof. Let $D_{i}$ be the domain of $\mathcal{F}_{i}$ and $D=\sum D_{i}$ the domain of $\mathcal{F}$. Define for $i \in I$ the mapping $\psi_{i}: \mathcal{P}(D) \rightarrow \mathcal{P}\left(D_{i}\right)$ by

$$
\psi_{i}(M)=\left\{a \in D_{i}: \forall j \geq i \forall b \in \pi_{i, j}^{-1}(a), b \in M\right\} .
$$

For every $a \in D_{i}$ the set $\left\{M: a \in \psi_{i}(M)\right\}$ is $\Pi_{1}^{0}$. Thus $\psi_{i}$ is of Baire class 1 . Moreover, a set $M$ is in $\mathcal{F}$ if and only if there are $i$ and $N \subset D_{i}$ in $\mathcal{F}_{i}$ such that $M \supset \hat{N}$, i.e. $\psi_{i}(M) \in \mathcal{F}_{i}$. Finally, since $\psi_{i}^{-1}\left(\mathcal{F}_{i}\right) \in \boldsymbol{\Sigma}_{\eta_{i}+1}^{0} \subset \boldsymbol{\Sigma}_{\xi}^{0}$, we have $\mathcal{F}=\bigcup_{i \in I} \psi_{i}^{-1}\left(\mathcal{F}_{i}\right) \in \Sigma_{\xi}^{0}$.

Proposition 5.8. Any quasi-inductive system $\left(\mathcal{F}_{i}, \pi_{j, i}\right)_{i \leq j, i, j \in I}$ of filters admits a canonical extension to a quasi-inductive system $\left(\mathcal{F}_{i}, \pi_{j, i}\right)_{i \leq j, i, j \in \hat{I}}$ of filters, where $\hat{I}=I \cup\{\infty\}$ is the directed set obtained by adding to $I$ a largest element $\infty$, and $\mathcal{F}_{\infty}$ is the Fubini sum of the family $\left(\mathcal{F}_{i}\right)_{i \in I}$.

Proof. For all $i \in I$ set $\pi_{\infty, i}=\sum_{j \geq i, i, j \in I} \pi_{j, i}$ (the disjoint union mapping): one easily checks that this defines a quasi-homomorphism from $\mathcal{F}_{\infty}$ to $\mathcal{F}_{i}$ and that the extended system $\left(\pi_{j, i}\right)_{i \leq j, i, j \in \hat{I}}$ is coherent.

6. Some canonical filters. Our goal in this section is to specify a canonical family $\left(\mathcal{N}_{\xi}\right)_{\xi<\omega_{1}}$ of Borel filters such that for each $\xi$ the filter $\mathcal{N}_{\xi}$ generates the Borel class $\mathcal{B}_{\xi}$, hence is of rank $\xi$. We recall that a similar family was already constructed by Katětov for the generation of the Baire classes $\mathcal{B}^{(\xi)}$, which we recall next. 
6.1. The Katětov filters $\mathcal{N}^{(\xi)}$. Consider the family $\left(\mathcal{N}^{(\xi)}\right)_{\xi<\omega_{1}}$ of filters defined inductively by:

$$
\left\{\begin{array}{l}
\left.\mathcal{N}^{(0)}=\{\{0\}\} \text { (the unique filter on }\{0\}\right), \\
\mathcal{N}^{(\xi+1)} \text { is the Fréchet extension of } \mathcal{N}^{(\xi)}, \\
\mathcal{N}^{(\lambda)} \text { is the Fubini sum of the family }\left(\mathcal{N}^{(\xi)}\right)_{\xi<\lambda}, \text { if } \lambda \text { is limit. }
\end{array}\right.
$$

Theorem 6.2 (Katětov). For all $\xi$, the filter $\mathcal{N}^{(\xi)}$ generates the Baire class $\mathcal{B}^{(\xi)}$.

This result is a particular case of Theorem 6.5 below. We point out that the proof of Theorem 6.5 will be based on totally different arguments than Katětov's original proof of Theorem 6.2.

6.2. The filters $\mathcal{N}_{\xi}$. Since the Baire class $\mathcal{B}^{(1+\xi)}$ is just the Borel class $\mathcal{B}_{\xi+1}$ we shall make a shift in the notations and set

$$
\mathcal{N}_{0}=\mathcal{N}^{(0)}, \quad \mathcal{N}_{\xi+1}=\mathcal{N}^{(1+\xi)} .
$$

To define $\mathcal{N}_{\lambda}$ for $\lambda$ limit, observe that since in the Katětov family $\left(\mathcal{N}^{(\xi)}\right)_{\xi<\omega_{1}}$ each $\mathcal{N}^{(\xi)}$ is obtained as the Fubini sum of the previous filters, applying Proposition 5.8 inductively one gets a coherent system $\left(\pi_{\eta, \xi}\right)_{\eta<\xi<\lambda}$ of quasihomomorphisms for this family, hence $\left(\mathcal{N}^{(\xi)}, \pi_{\eta, \xi}\right)_{\eta<\xi<\lambda}$ is a quasi-inductive system of filters and we can define

$$
\mathcal{N}_{\lambda}=\lim _{\overleftarrow{\xi<\lambda}} \mathcal{N}^{(\xi)}
$$

Proposition 6.4. For all $\xi$, the filter $\mathcal{N}_{\xi}$ is Borel. More precisely:

(a) If $\xi=\lambda+n$ with $\lambda$ limit and $1 \leq n<\omega$ then $\mathcal{N}_{\xi}$ is a $\Sigma_{1+\lambda+2 n-1}^{0}$ set.

(b) If $\lambda$ is limit then $\mathcal{N}_{\lambda}$ is a $\boldsymbol{\Sigma}_{\lambda}^{0}$ set.

Proof. It follows by a straightforward induction from Proposition 4.6 that if $n$ is finite then $\mathcal{N}_{n}$ is a $\boldsymbol{\Sigma}_{2 n}^{0}$ set, and if moreover $\lambda$ is limit then $\mathcal{N}_{\lambda+n+1}=\mathcal{N}^{(\lambda+n)}$ is a $\boldsymbol{\Sigma}_{\lambda+2 n+1}^{0}$ set. This proves (a).

(b) follows from (a) and Proposition 5.7.

Theorem 6.5. For all $\xi$, the filter $\mathcal{N}_{\xi}$ generates the Borel class $\mathcal{B}_{\xi}$.

Proof. It follows from the inductive construction of the Katětov filters $\mathcal{N}^{(\xi)}$ and Proposition 4.4(a) and (c) that $\operatorname{rk}\left(\mathcal{N}^{(1+\xi)}\right)=\xi+1$. Moreover, if $\lambda$ is limit then by Proposition 5.5 we have $\lambda \leq \operatorname{rk}\left(\mathcal{N}_{\lambda}\right) \leq \lambda+1$; and since by Proposition 6.4 the filter $\mathcal{N}_{\lambda}$ is itself a $\Sigma_{\lambda}^{0}$ set, it follows from Proposition 5.5 that $\operatorname{rk}\left(\mathcal{N}_{\lambda}\right)=\lambda$.

Hence $\operatorname{rk}\left(\mathcal{N}_{\xi}\right)=\xi$ for all $\xi$, and it follows from Theorem 2.6 that for any Polish space $X$ we have $\mathcal{C}_{\mathcal{N}_{\xi}}(X) \subset \mathcal{B}_{\xi}$. Notice that by Theorem 2.8 if $X$ is 
zero-dimensional then the converse inclusion $\mathcal{C}_{\mathcal{N}_{\xi}}(X) \supset \mathcal{B}_{\xi}$ also holds, but we have to prove it for an arbitrary Polish space.

We first treat the successor case $\xi+1$. Observe that by the LebesgueHausdorff Theorem any function $f: X \rightarrow \mathbb{R}$ of Baire class $\mathcal{B}^{(\xi)}$ is the pointwise limit of a sequence $\left(f_{k}\right)$ of functions of Baire class $<\xi$; and if each $f_{k}$ itself is the limit along a filter $\mathcal{F}_{k}$ of continuous functions $\left(f_{k, n}\right)$ then clearly $f$ itself is the limit of $\left(f_{k, n}\right)$ along the Fubini sum of the $\mathcal{F}_{k}$. It follows by straightforward induction on $\xi$ that such a function $f$ is the limit along $\mathcal{N}^{(\xi)}=\mathcal{N}_{\xi+1}$ of the family $\left(f_{k, n}\right)$. Hence $\mathcal{C}_{\mathcal{N}_{\xi+1}}(X) \supset \mathcal{B}_{\xi+1}$.

Suppose now that $\lambda$ is limit and observe that if $A$ is any $\boldsymbol{\Pi}_{\xi}^{0}$ subset of $X$ for some $\xi<\lambda$, then the function $\mathbb{1}_{A}$ is of Baire class $<\lambda$. Thus $\mathbb{1}_{A}$ is the limit of continuous functions along $\mathcal{N}_{\eta}$ for $\xi<\eta<\lambda$, hence the limit of continuous functions along $\mathcal{N}_{\lambda}$. Then by Lemmas 2.1 and 2.2, $\mathcal{C}_{\mathcal{N}_{\lambda}}(X) \supset \mathcal{B}_{\lambda}$.

REMARK 6.6. We mentioned in the introduction that in [9] Louveau constructs a unique $\Pi_{1}^{1}$ filter $\mathcal{F}$ which generates the whole class $\mathcal{B}$. In fact, this filter is provided with a natural $\Pi_{1}^{1}$ norm $\rho: \mathcal{F} \rightarrow \omega_{1}$ with the property that for all $\xi<\omega_{1}$ the set $\mathcal{F}_{\xi}:=\{M \in \mathcal{F}: \rho(M)<\xi\}$ is actually a filter; and a careful analysis of the norm $\rho$ shows that the filter $\mathcal{F}_{\xi}$ is also of rank $\xi$ and has exactly the same Borel complexity as $\mathcal{N}_{\xi}$.

\section{A dichotomy for $\Sigma_{1}^{1}$ filters}

Notation 7.1. Given two filters $\mathcal{F}$ and $\mathcal{G}$ we shall write $\mathcal{F} \sqsubseteq \mathcal{G}$ if there exists a bijection $\sigma: \operatorname{dom}(\mathcal{F}) \rightarrow \operatorname{dom}(\mathcal{G})$ which sends any element of $\mathcal{F}$ onto an element of $\mathcal{G}$.

Lemma 7.2. If $\mathcal{F} \sqsubseteq \mathcal{G}$ then $\operatorname{rk}(\mathcal{F}) \leq \operatorname{rk}(\mathcal{G})$.

Proof. Let $\xi=\operatorname{rk}(\mathcal{G})$ and $S$ be a $\boldsymbol{\Sigma}_{1+\xi}^{0}$ subset of $\mathcal{P}(\operatorname{dom}(\mathcal{G}))$ separating $\mathcal{G}$ from $\mathcal{G}^{*}$. Since $\sigma$ is one-to-one, the mapping $M \mapsto \sigma(M)$ is continuous from $\mathcal{P}(\operatorname{dom}(\mathcal{F}))$ to $\mathcal{P}(\operatorname{dom}(\mathcal{G}))$ and the set $S^{\prime}=\{M: \sigma(M) \in S\}$ is $\boldsymbol{\Sigma}_{1+\xi}^{0}$ in $\mathcal{P}(\operatorname{dom}(\mathcal{F}))$ and separates $\mathcal{F}$ from $\mathcal{F}^{*}$. Hence $\operatorname{rk}(\mathcal{F}) \leq \xi$.

Proposition 7.3. A filter $\mathcal{F}$ is of rank $\geq 1$ if and only if $\mathcal{N}_{1} \sqsubseteq \mathcal{F}$.

Proof. If $\mathcal{N}_{1} \sqsubseteq \mathcal{F}$ we have $1=\operatorname{rk}\left(\mathcal{N}_{1}\right) \leq \operatorname{rk}(\mathcal{F})$. Conversely, if $\operatorname{rk}(\mathcal{F})>0$ then $\mathcal{F}$ is free by Proposition 3.3, and if $\sigma$ is any bijection from $\omega$ to $\operatorname{dom}(\mathcal{F})$, it is easy to see that for each cofinite subset $M$ of $\omega, \sigma(M)$ is cofinite in $\operatorname{dom}(\mathcal{F})$, hence in $\mathcal{F}$. So $\mathcal{N}_{1} \sqsubseteq \mathcal{F}$.

Lemma 7.4. Let $D$ be a countable set, $\mathcal{F}$ a filter on $D$, and $\left(M_{i}\right)_{i \in I}$ a countable family of elements of $\mathcal{F}^{*}$ with the property

$$
\forall M \subset D\left(\forall i \in I, M \cap M_{i} \text { is finite } \Rightarrow M \in \mathcal{F}^{*}\right) \text {. }
$$

Then $\mathcal{N}_{2} \sqsubseteq \mathcal{F}$. 
Proof. Without loss of generality we can assume $I=\omega$. Replacing if necessary $M_{i}$ by $M_{i}^{\prime}=M_{i} \backslash \bigcup_{j<i} M_{j}$ we can also assume that the $M_{i}$ 's are pairwise disjoint: indeed, if $M \cap M_{i}^{\prime}$ is finite for all $i$, then so is $M \cap M_{i} \subset$ $\bigcup_{j \leq i} M \cap M_{j}^{\prime}$ for all $i$.

Let $N_{0}=D \backslash \bigcup_{i \in I} M_{i}=D \backslash \bigcup_{i \in I} M_{i}^{\prime}$. Since $N_{0} \cap M_{i}=\emptyset$ for all $i$, it follows clearly from $(\star)$ that $N_{0} \in \mathcal{F}^{*}$. If $J=\left\{i: M_{i}^{\prime}\right.$ is finite $\}$ we see in the same way that $N_{1}=\bigcup_{i \in J} M_{i}^{\prime} \in \mathcal{F}^{*}$ and that $I^{\prime}:=I \backslash J$ is infinite. Choose $i_{0} \in I^{\prime}=I \backslash J$ and define, for $i \in I^{\prime}$,

$$
M_{i}^{\prime \prime}= \begin{cases}M_{i}^{\prime} & \text { if } i \neq i_{0}, \\ M_{i_{0}}^{\prime} \cup N_{0} \cup N_{1} & \text { if } i=i_{0} .\end{cases}
$$

Then $\left(M_{i}^{\prime \prime}\right)_{i \in I^{\prime}}$ is a countable partition of $D$ into infinite sets still satisfying $(\star)$. Choose a bijection $f$ from $\omega$ onto $I^{\prime}$ and for each integer $p$ a bijection $g_{p}$ from $\omega$ onto $M_{f(p)}^{\prime \prime}$. Then define a bijection $\sigma$ from $\omega^{2}=\operatorname{dom}\left(\mathcal{N}_{2}\right)$ onto $D$ by $\sigma(p, q)=g_{p}(q)$. If $A \in \mathcal{N}_{2}^{*}$ there is an $m$ such that $A_{p}:=\{q:(p, q) \in A\}$ is finite for all $p>m$. Then $M^{\prime}=\bigcup_{p>m} g_{p}\left(A_{p}\right) \in \mathcal{F}^{*}$ by $(\star)$, and so is $\sigma(A) \subset M^{\prime} \cup \bigcup_{p \leq m} M_{f(p)}^{\prime \prime}$. This shows that $\mathcal{N}_{2} \sqsubseteq \mathcal{F}$.

Theorem 7.5. A $\boldsymbol{\Sigma}_{1}^{1}$ filter $\mathcal{F}$ is of rank $\geq 2$ if and only if $\mathcal{N}_{2} \sqsubseteq \mathcal{F}$.

Proof. If $\mathcal{N}_{2} \sqsubseteq \mathcal{F}$, we have $2=\operatorname{rk}\left(\mathcal{N}_{2}\right) \leq \operatorname{rk}(\mathcal{F})$.

Conversely, if $\operatorname{rk}(\mathcal{F}) \geq 2$ we have $\mathcal{C}_{\mathcal{F}}\left(\mathbf{2}^{\omega}\right) \supset \mathcal{B}_{2}\left(\mathbf{2}^{\omega}\right)$. Denote by $D$ the domain of $\mathcal{F}$, and by $\mathbb{Q}$ the subspace $\left\{\alpha \in \mathbf{2}^{\omega}: \exists p \forall q \geq p, \alpha(q)=0\right\}$ of $\mathbf{2}^{\omega}$, which is countable and dense. Then $\mathbb{1}_{\mathbb{Q}} \in \mathcal{B}_{2}\left(\mathbf{2}^{\omega}\right)$ and we can find a sequence $\left(f_{n}\right)_{n \in D}$ of continuous functions $\mathbf{2}^{\omega} \rightarrow\{0,1\}$ such that $\mathbb{1}_{\mathbb{Q}}=\lim _{\mathcal{F}} f_{n}$.

For every $\alpha \in 2^{\omega}$ denote by $M(\alpha)$ the set $\left\{n \in D: f_{n}(\alpha)=0\right\}$. We have $M(\alpha) \in \mathcal{F}^{*}$ if $\alpha \in \mathbb{Q}$, and $M(\alpha) \in \mathcal{F}$ if $\alpha \notin \mathbb{Q}$. We claim that the family $(M(\alpha))_{\alpha \in \mathbb{Q}}$ has property $(\star)$ from Lemma 7.4, which is enough for proving the theorem. Indeed, assume that $M \in \mathcal{P}(D)$ has finite intersection with all $M(\alpha)$ for $\alpha \in \mathbb{Q}$.

We want to construct $\beta, \gamma \in 2^{\omega} \backslash \mathbb{Q}$ such that $N=M \cap M(\beta) \cap M(\gamma)$ is finite, and this will imply that $M \subset N \cup(D \backslash M(\beta)) \cup(D \backslash M(\gamma)) \in \mathcal{F}^{*}$.

Define $\mathbf{0} \in 2^{\omega}$ to be the infinite null sequence $(\mathbf{0}(n)=0$ for all $n), N$ to be the finite set $M \cap M(\mathbf{0})$, and $u_{n}$ to be the sequence of length $n+1$ having $n$ zeros followed by one 1 . We construct, by induction on the integer $k$, sequences $s_{k}$ and $t_{k}$ in $2^{<\omega}$ such that $M \cap M\left(s_{k} \frown \mathbf{0}\right) \cap M\left(t_{k} \frown \mathbf{0}\right)=N$. Put $s_{0}=t_{0}=\emptyset$. Observe that the function $\alpha \mapsto M(\alpha)$ is continuous and that $\lim _{n \rightarrow \infty} s_{k} \frown u_{n} \frown \mathbf{0}=s_{k} \frown \mathbf{0}$. Thus since $M \cap M\left(t_{k} \frown \mathbf{0}\right)$ is finite and $M\left(s_{k} \frown u_{n} \frown \mathbf{0}\right) \rightarrow M\left(s_{k} \frown \mathbf{0}\right)$, there is some $n_{k}$ large enough such that

$$
M \cap M\left(t_{k} \frown \mathbf{0}\right) \cap M\left(s_{k} \frown u_{n_{k}} \frown \mathbf{0}\right)=M \cap M\left(t_{k} \frown \mathbf{0}\right) \cap M\left(s_{k} \frown \mathbf{0}\right)=N,
$$

and we define $s_{k+1}=s_{k} \frown u_{n_{k}}$. In the same way, since $M \cap M\left(s_{k+1} \frown \mathbf{0}\right)$ is 
finite there is $m_{k}$ large enough such that

$$
M \cap M\left(s_{k+1} \frown \mathbf{0}\right) \cap M\left(t_{k} \frown u_{m_{k}} \frown \mathbf{0}\right)=M \cap M\left(s_{k+1} \frown \mathbf{0}\right) \cap M\left(t_{k} \frown \mathbf{0}\right)=N,
$$

and we define $t_{k+1}=t_{k} \frown u_{m_{k}}$. Then there are $\beta$ and $\gamma$ in $2^{\omega} \backslash \mathbb{Q}$ such that $s_{k} \prec \beta$ and $t_{k} \prec \gamma$ for all $k$. It is clear that $s_{k} \frown \mathbf{0} \rightarrow \beta$ and $t_{k} \frown \mathbf{0} \rightarrow \gamma$. Thus by continuity we get

$$
M \cap M(\beta) \cap M(\gamma)=\lim _{k \rightarrow \infty} M \cap M\left(s_{k} \frown \mathbf{0}\right) \cap M\left(t_{k} \frown \mathbf{0}\right)=N,
$$

and this completes the proof.

Corollary 7.6. If $\mathcal{F}$ is a $\boldsymbol{\Sigma}_{1}^{1}$ filter of rank $\geq 2$ then $\mathcal{C}_{\mathcal{F}}(X) \supset \mathcal{B}_{2}(X)$ for any Polish space $X$.

Proof. Since $\mathcal{N}_{2} \sqsubseteq \mathcal{F}$ there exists a bijection $\sigma$ from $\omega^{2}=\operatorname{dom}\left(\mathcal{N}_{2}\right)$ to $\operatorname{dom}(\mathcal{F})$ such that $\sigma(A) \in \mathcal{F}$ for all $A \in \mathcal{N}_{2}$. If $X$ is a Polish space and $f: X \rightarrow \mathbb{R}$ is of Baire class 2 , there is a family $\left(f_{p, q}\right)_{(p, q) \in \omega^{2}}$ of continuous functions $X \rightarrow \mathbb{R}$ such that $f=\lim _{p \rightarrow \infty} \lim _{q \rightarrow \infty} f_{p, q}$. It follows that $f=$ $\lim _{\mathcal{N}_{2}} f_{p, q}$, and hence $f=\lim _{\mathcal{F}} g_{n}$ where $g_{n}=f_{p, q}$ with $(p, q)=\sigma^{-1}(n)$.

This shows that $f \in \mathcal{C}_{\mathcal{F}}(X)$.

Corollary 7.7. If $\mathcal{F}$ is a $\boldsymbol{\Sigma}_{1}^{1}$ filter then one and only one of the following two alternatives holds: either

- $\mathcal{C}_{\mathcal{F}}(X) \subset \mathcal{B}_{1}(X)$ for any Polish space $X$, or

- $\mathcal{N}_{2} \sqsubseteq \mathcal{F}$.

Proof. By Theorem 2.6 the first alternative is equivalent to $\operatorname{rk}(\mathcal{F}) \leq 1$, and by Theorem 7.5 the second one is equivalent to $\operatorname{rk}(\mathcal{F}) \geq 2$.

Proposition 7.3 and Theorem 7.5 suggest the following conjecture for any countable ordinal $\xi$.

Conjecture 7.8. A $\boldsymbol{\Sigma}_{1}^{1}$ filter $\mathcal{F}$ is of rank $\geq \xi$ if and only if $\mathcal{N}_{\xi} \sqsubseteq \mathcal{F}$.

8. Comparing the rank and the class of a Borel filter. Given a Borel set one is naturally interested in finding its "exact" complexity. For this the Wadge hierarchy provides a complete scale of comparison, but here we restrict this scale to the more classical Borel hierarchy by considering only the additive and multiplicative Borel classes. For such a class $\boldsymbol{\Gamma}$ we denote as usual by $\check{\boldsymbol{\Gamma}}$ its dual class. We recall that by the Wadge Theorem a set $A$ is in $\boldsymbol{\Gamma} \backslash \check{\boldsymbol{\Gamma}}$ if and only if $A$ is $\boldsymbol{\Gamma}$-complete (see [7]). When we say that a Borel set $A$ is of class $\xi$ with no more specification we mean that $A$ is in $\Sigma_{\xi}^{0}$ or in $\Pi_{\xi}^{0}$.

The aim of this section is to discuss the relations between the rank of a Borel filter and its Borel class.

Proposition 8.1. For any ordinals $\xi, \eta$ there exists a Borel filter of rank $\xi$ and class $\geq \eta$. 
Proof. Without loss of generality we may assume that $\eta \geq \xi$. Set $D_{\xi}:=$ $\operatorname{dom}\left(\mathcal{N}_{\xi}\right), D_{\eta}:=\operatorname{dom}\left(\mathcal{N}_{\eta}\right)$ and consider on $D:=D_{\xi}+D_{\eta}$ the filter $\mathcal{F}$ of all subsets $M$ such that $M \cap \operatorname{dom}\left(\mathcal{N}_{\xi}\right) \in \mathcal{N}_{\xi}$ and $M \cap \operatorname{dom}\left(\mathcal{N}_{\eta}\right) \in \mathcal{N}_{\eta}$.

If $\mathcal{G}_{0} \subset \mathcal{P}\left(\operatorname{dom}\left(\mathcal{N}_{\xi}\right)\right)$ is any $\boldsymbol{\Sigma}_{1+\xi}^{0}$ set separating the filter $\mathcal{N}_{\xi}$ from its ideal then the set $\mathcal{G}=\left\{M \subset D: M \cap D_{\xi} \in \mathcal{G}_{0}\right\}$ is $\boldsymbol{\Sigma}_{1+\xi}^{0}$ too and $\mathcal{G}$ separates $\mathcal{F}$ from its ideal, hence $\operatorname{rk}(\mathcal{F}) \leq \xi$.

To see that $\operatorname{rk}(\mathcal{F})=\xi$ it is enough to prove that any function $f$ in $\mathcal{B}_{\xi}\left(\mathbf{2}^{\omega}\right)$ is limit along $\mathcal{G}$ of continuous functions on $\mathbf{2}^{\omega}$. Since $\mathcal{N}_{\xi}$ generates $\mathcal{B}_{\xi}$, and $\mathcal{N}_{\eta}$ generates $\mathcal{B}_{\eta} \supset \mathcal{B}_{\xi}$, there are continuous functions $f_{n}: \mathbf{2}^{\omega} \rightarrow \mathbb{R}$ for $n \in D_{\xi}$ and $f_{m}: \mathbf{2}^{\omega} \rightarrow \mathbb{R}$ for $m \in D_{\eta}$ such that $f=\lim _{\mathcal{N}_{\xi}} f_{n}=\lim _{\mathcal{N}_{\eta}} f_{m}$. Putting together these two families we get a family $\left(f_{i}\right)_{i \in D}$ such that $f=\lim _{\mathcal{G}} f_{i}$.

Finally, consider the continuous mapping $\Psi: M \mapsto D_{\xi}+M$ from $\mathcal{P}\left(D_{\eta}\right)$ to $\mathcal{P}(D)$. One easily checks $\Psi^{-1}(\mathcal{F})=\mathcal{N}_{\eta}$. Hence if $\mathcal{F}$ were of class $<\eta$ then so would be $\mathcal{N}_{\eta}$, which is impossible since $\mathcal{N}_{\eta}$ is of rank $\eta$. This proves that $\mathcal{F}$ is of class $\geq \eta$.

In fact, for small ranks (0 or 1$)$ we even have the following more precise result.

Proposition 8.2. If $i=0$ or 1 then for any Borel class $\boldsymbol{\Gamma} \supset \boldsymbol{\Sigma}_{2}^{0}$ there exists in $\boldsymbol{\Gamma} \backslash \check{\boldsymbol{\Gamma}}$ a filter of rank $i$.

Proof. Observe first that the case $i=0$ follows from the case $i=1$. To see this observe that if $\mathcal{F}$ is any free filter and $a$ is any element of its domain then the non-free filter $\mathcal{F}(a):=\{M \in \mathcal{F}: a \in M\}$ is of rank 0. Moreover, $\mathcal{F}(a)$ is a $\boldsymbol{\Delta}_{1}^{0}$ subset of $\mathcal{F}$ and $\mathcal{F}=\Phi^{-1}(\mathcal{F}(a))$ where $\Phi: M \mapsto M \cup\{a\}$ is a continuous mapping. It follows from these observations that $\mathcal{F}$ is in some given class $\boldsymbol{\Gamma}$ if and only $\mathcal{F}(a)$ is in $\boldsymbol{\Gamma}$.

So we now suppose that $i=1$ and we treat separately the cases where $\boldsymbol{\Gamma}$ is additive or multiplicative. Observe that since $\boldsymbol{\Gamma} \supset \boldsymbol{\Sigma}_{2}^{0}$, in the first case $\boldsymbol{\Gamma}=\boldsymbol{\Sigma}_{\xi}^{0}$ with $\xi \geq 2$, so $\boldsymbol{\Gamma}$ is at least of class 2 .

We first treat the case where $\boldsymbol{\Gamma}$ is additive, and for this we shall make use of a family of filters considered by Lutzer, van Mill and Pol in [12]: For any $x \in \mathbf{2}^{\omega}$ set $M_{x}=\left\{s \in 2^{<\omega}: s \prec x\right\}$; then given any non-empty Borel subset $A$ of $2^{\omega}$ consider on the countable set $2^{<\omega}$ the ideal $\mathcal{I}_{\mathcal{A}}$ generated by the set $\left\{M_{x} \in \mathcal{P}\left(2^{<\omega}\right): x \in A\right\}$ and let $\Phi_{A}=\mathcal{I}_{A}^{*}$ be its dual filter. The exact complexity of these filters was computed by Calbrix in [2], and it turns out that for $\xi \geq 2, \Phi_{A}$ is $\boldsymbol{\Sigma}_{\xi}^{0}$ if and only if $A$ itself is $\boldsymbol{\Sigma}_{\xi}^{0}$.

Observe that since $\Phi_{A} \subset \Phi_{2^{\omega}}$, we have $\operatorname{rk}\left(\Phi_{A}\right) \leq \operatorname{rk}\left(\Phi_{\mathbf{2}^{\omega}}\right)$. Moreover, since $\mathcal{I}_{\mathbf{2}^{\omega}}$ is generated by the compact set $\left\{M_{x} \in \mathcal{P}\left(2^{<\omega}\right): x \in \mathbf{2}^{\omega}\right\}$, it follows that $\mathcal{I}_{\mathbf{2}^{\omega}}$ is $\boldsymbol{\Sigma}_{2}^{0}$, hence so is $\Phi_{\mathbf{2}^{\omega}}=\mathcal{I}_{A}^{*}$, and Theorem 2.6 shows that $\operatorname{rk}\left(\Phi_{2^{\omega}}\right) \leq 1$. This proves that $\operatorname{rk}\left(\Phi_{A}\right) \leq 1$ for all $A$, and since this filter is clearly free, we have $\operatorname{rk}\left(\Phi_{A}\right)=1$. Hence for all $\xi \geq 2$ if we set $\mathcal{F}_{\xi}:=\Phi_{A_{\xi}}$ 
where $A_{\xi}$ is some $\boldsymbol{\Sigma}_{\xi}^{0}$-complete set, it follows from the previous observations that $\mathcal{F}_{\xi}$ is $\boldsymbol{\Sigma}_{\xi}^{0}$-complete and of rank 1 .

For the case where $\boldsymbol{\Gamma}$ is multiplicative fix for all $\xi \geq 3$ a sequence $\left(\xi_{p}\right)_{p \in \omega}$ such that $\xi=\sup _{p}\left(\xi_{p}+1\right)$ and let $\mathcal{G}_{\xi}$ be the complete filter sum of the family $\left(\mathcal{F}_{\xi_{p}}\right)_{p \in \omega}$. Then by standard arguments one can prove that each $\mathcal{G}_{\xi}$ is $\boldsymbol{\Pi}_{\xi}^{0}$-complete and it follows from Proposition 4.5 that $\mathcal{G}_{\xi}$ is of rank 1.

We now consider the inverse problem of seeking "simple" filters of some given rank $\xi$.

Notation 8.3. For all $\xi>0$, if $\xi=\lambda+n$ with $\lambda$ limit and $n<\omega$ we set

$$
\xi^{*}= \begin{cases}\lambda & \text { if } \xi=\lambda \text { is limit, } \\ 1+\lambda+2 n-1 & \text { if } \xi \text { is successor. }\end{cases}
$$

In particular, if $\xi=n$ is finite then $\xi^{*}=2 n$.

Summing up the conclusions of Corollary 2.10, Proposition 6.4 and Theorem 6.5 we can state:

Theorem 8.4. For all $\xi>0$ :

(a) There is no $\boldsymbol{\Pi}_{1+\xi}^{0}$ filter of rank $\geq \xi$.

(b) There exists a $\boldsymbol{\Sigma}_{\xi^{*}}^{0}$ filter of rank $\xi$.

Observe that when $\xi^{*}>1+\xi$ Theorem 8.4 does not give any information about the possible existence of a $\boldsymbol{\Pi}_{\eta}^{0}$ filter of rank $\xi$ with $\eta \leq \xi^{*}$. In fact, we conjecture that there is none:

Conjecture 8.5. There is no $\boldsymbol{\Pi}_{\xi^{*}}^{0}$ filter of rank $\geq \xi$.

REMARK 8.6. If $\xi=\lambda>0$ is limit then $\xi^{*}=\lambda=1+\xi$. Similarly if $\xi=\lambda+1$ with $\lambda>0$ limit then $\xi^{*}=\lambda+1=1+\xi$. Hence in both cases the conjecture is just a restatement of part (a) of Theorem 8.4.

Thus the conjecture concerns only successor ranks. Observe also that if $\xi=1$ then $\xi^{*}=2$ and the conjecture is also true, since by Proposition 3.3 a filter of rank 1 is free, and it is a classical fact that such a filter is necessarily meagre and cannot be a $\boldsymbol{\Pi}_{2}^{0}$ set.

9. Borel class of filters of rank $\geq 2$. The aim of this section is to prove Conjecture 8.5 for $\xi=2$, in which case $\xi^{*}=4$. Notice Theorem 8.4 ensures already that a $\Pi_{3}^{0}$ filter is of rank $\leq 1$.

Theorem 9.1. Any $\boldsymbol{\Pi}_{4}^{0}$ filter is of rank $\leq 1$.

In fact, Theorem 9.1 will follow from some general combinatorial result on filters of rank $\geq 2$. Before we state this result let us first point out a simple consequence of Theorem 2.6, which can also be derived from Theorem 1.4 of $[15]$. 
In all what follows, when we speak about the intersection of some elements of a set $\mathcal{G} \subset \mathcal{P}(\omega)$ we shall always be implicitly referring to the intersection operation in $\mathcal{P}(\omega)$.

TheOREM 9.2. Let $\mathcal{F}$ be a $\boldsymbol{\Sigma}_{1}^{1}$ filter of rank $\geq 2$. Then any $\Pi_{3}^{0}$ set $\mathcal{G} \supset \mathcal{F}$ contains two elements with empty intersection.

Proof. Observe that if $\mathcal{G}$ is a $\Pi_{3}^{0}$ set then the set $\mathcal{G} \cap \mathcal{G}^{*}$ is necessarily non-empty, for otherwise, by Lemma $1.4, \mathcal{F}$ would be $\boldsymbol{\Delta}_{3}^{0}$-separated from $\mathcal{F}^{*}$ and, by Theorem 2.6, $\mathcal{F}$ would then be of rank $<2$. Now if $M$ is any element of $\mathcal{G} \cap \mathcal{G}^{*}$ then $M$ and $M^{c}$ are two disjoint elements of $\mathcal{G}$.

We now prove a $\boldsymbol{\Pi}_{4}^{0}$ version of this result, from which Theorem 9.1 follows readily.

TheOREM 9.3. Let $\mathcal{F}$ be a $\boldsymbol{\Sigma}_{1}^{1}$ filter of rank $\geq 2$. Then any $\boldsymbol{\Pi}_{4}^{0}$ set $\mathcal{G} \supset \mathcal{F}$ contains three elements with empty intersection.

Proof. By Theorem 7.5 we may and shall assume that $\mathcal{F}$ is the canonical filter $\mathcal{N}_{2}$ on $\omega^{2}$. So let $\mathcal{G}$ be a $\Pi_{4}^{0}$ set containing $\mathcal{F}=\mathcal{N}_{2}$.

Our goal is to construct three subsets $M^{(0)}, M^{(1)}, M^{(2)}$ of $\omega^{2}$ in $\mathcal{G}$ such that $\bigcap_{i=0}^{2} M^{(i)}=\emptyset$. In fact, what we shall really and concretely construct are the characteristic functions $x^{(i)} \in \mathbf{2}^{\omega^{2}}$ of these sets, which will be defined in the form $x^{(i)}=\bigcup_{n} c_{n}^{(i)}$ with $c_{n}^{(i)} \in \mathbf{2}^{C_{n}}$ for some increasing sequence $\left(C_{n}\right)$ of infinite subsets of $\omega^{2}$ which will be defined inductively. For these reasons it will be important to avoid a systematic identification between $\mathcal{P}\left(\omega^{2}\right)$ and $\mathbf{2}^{\omega^{2}}$.

The construction is rather technical and necessitates the introduction of a number of preliminary notations.

We first set

$$
F=\left\{x \in \mathbf{2}^{\omega^{2}}:[x=1] \in \mathcal{F}\right\} \quad \text { and } \quad G=\left\{x \in \mathbf{2}^{\omega^{2}}:[x=1] \in \mathcal{G}\right\} .
$$

Since $G$ is a $\Pi_{4}^{0}$ set we can fix a family $\left(G_{(k, l, m)}\right)_{(k, l, m) \in \omega^{3}}$ of open subsets of $\mathbf{2}^{\omega^{2}}$ such that $G=\bigcap_{k} \bigcup_{l} \bigcap_{m} G_{(k, l, m)}$. We also set $G_{(k)}=\bigcup_{l} \bigcap_{m} G_{(k, l, m)}$ for all $k$, and $G_{(k, l)}=\bigcap_{m} G_{(k, l, m)}$ for all $l$; and we shall assume that the sequence $\left(G_{(k)}\right)_{k \in \omega}$ is decreasing.

We denote by $\pi: \omega^{2} \rightarrow \omega$ the canonical projection on the first factor. We shall say that a subset $M$ of $\omega^{2}$ is a cylinder if it is of the form $M=M_{0} \times \omega$ with $M_{0}=\pi(M)$ finite. We denote by $\mathcal{C}$ the set of all cylinders.

We denote by $\Omega$ the set of all partial mappings from $\omega^{2}$ to $\{0,1\}$. If $a \in \Omega$ we denote as usual by $\operatorname{dom}(a)$ its domain. We consider on $\Omega$ the standard extension (inclusion) relation $\subset$.

We also consider on $\Omega$ the pointwise product operation: if $a, b \in \Omega$ then by definition $\operatorname{dom}(a \cdot b)=\operatorname{dom}(a) \cap \operatorname{dom}(b)$ and $a \cdot b(p)=a(p) \cdot b(p)$ for any $p=\left(p_{0}, p_{1}\right) \in \operatorname{dom}(a . b)$. We denote by $\mathbf{0}$ the constant mapping on $\omega^{2}$ with 
value 0 ; so $a . b \subset \mathbf{0}$ is equivalent to $[a=1] \cap[b=1]=\emptyset$. Similarly we denote by $\mathbb{1}$ the constant mapping on $\omega^{2}$ with value 1 . For any $a \in \Omega$ we set

$$
N_{a}=\left\{x \in \mathbf{2}^{\omega^{2}}: a \subset x\right\} .
$$

Notice that if $\operatorname{dom}(a)$ is finite then $N_{a}$ is a clopen subset of $\mathbf{2}^{\omega^{2}}$ but in general $N_{a}$ is only closed.

Lastly, we introduce a subset $\Sigma$ of $\Omega \times \Omega$ which will play a crucial role in the proof and which is defined by

$$
(a, b) \in \Sigma \Leftrightarrow\left\{\begin{array}{l}
\operatorname{dom}(a) \text { is finite, } \\
\operatorname{dom}(b) \in \mathcal{C},[b=0] \text { is finite, } \\
\operatorname{dom}(a) \cap \operatorname{dom}(b)=\emptyset
\end{array}\right.
$$

We consider on $\Sigma$ the product ordering induced by $(\Omega, \subset)$ and that we also denote for simplicity by $\subset$ since there is no possible confusion. So

$$
(a, b) \subset\left(a^{\prime}, b^{\prime}\right) \Leftrightarrow a \subseteq a^{\prime} \text { and } b \subseteq b^{\prime} \Rightarrow a \cup b \subset a^{\prime} \cup b^{\prime} \Rightarrow b \subset b^{\prime}
$$

(the first equivalence is just the definition of a product ordering and the last implication follows from the conditions on the domains of $a$ and $b$ in the definition of $\Sigma$.)

Lemma 9.4. Let $E$ be any subset of $\mathbf{2}^{\omega^{2}}$ and $(a, b)$ in $\Sigma$. If for all $\left(a^{\prime}, b^{\prime}\right) \supset(a, b)$ in $\Sigma$ we have $N_{a^{\prime} \cup b^{\prime}} \cap E \neq \emptyset$ then $N_{a \cup b} \cap E$ is a dense subset of $N_{a \cup b}$.

Proof. Observe that if $c$ is any finite extension of $a \cup b$ then $c \backslash b$ is finite so $(c \backslash b, b) \in \Sigma$ and clearly $(c \backslash b, b) \supset(a, b)$, hence $N_{(c \backslash b) \cup b} \cap E=N_{c} \cap E \neq \emptyset$.

Lemma 9.5. Suppose that $\left(F_{l}\right)_{l \in \omega}$ is a countable covering of $F$. Then for all $(a, b)$ in $\Sigma$ there exist $l \in \omega$ and $\left(a^{\prime}, b^{\prime}\right) \supset(a, b)$ in $\Sigma$ such that $N_{a^{\prime \prime} \cup b^{\prime \prime}} \cap F_{l} \neq \emptyset$ for all $\left(a^{\prime \prime}, b^{\prime \prime}\right) \supset\left(a^{\prime}, b^{\prime}\right)$ in $\Sigma$.

Proof. Fix $(a, b) \in \Sigma$; consider the cylinder $A=\pi(\operatorname{dom}(a)) \times \omega$ and let $\Sigma^{\prime}:=\left\{\left(a^{\prime}, b^{\prime}\right) \in \Sigma:\left(a^{\prime}, b^{\prime}\right) \supset(a, b)\right.$ and $\left.\operatorname{dom}\left(a^{\prime}\right) \subset A\right\}$.

Observe that $(a, b) \in \Sigma^{\prime}$.

Claim. There exist $l \in \omega$ and $\left(a^{\prime}, b^{\prime}\right) \supset(a, b)$ in $\Sigma^{\prime}$ such that

$$
N_{a^{\prime \prime} \cup b^{\prime \prime}} \cap F_{l} \neq \emptyset \quad \text { for all }\left(a^{\prime \prime}, b^{\prime \prime}\right) \supset\left(a^{\prime}, b^{\prime}\right) \text { in } \Sigma^{\prime} .
$$

To prove the claim we proceed by contradiction: If not then starting from $(a, b)$ one constructs by straightforward induction an increasing sequence $\left(a_{l}, b_{l}\right)_{l \in \omega}$ in $\Sigma^{\prime}$ with the property that $N_{a_{l} \cup b_{l}} \cap F_{l}=\emptyset$ for each $l$. Let $c=\bigcup_{l}\left(a_{l} \cup b_{l}\right)$ and consider $x=c \cup \mathbb{1}_{\mid \omega^{2} \backslash \operatorname{dom}(c)} \in \mathbf{2}^{\omega^{2}}$.

Then $[x=0] \subset A \cup \bigcup_{l}\left[b_{l}=0\right]$ and from the definition of $\Omega$ this latter set is in the ideal $\mathcal{F}^{*}$, hence $[x=1] \in \mathcal{F}$ and so $x \in F$. But for all $l$, since $x \supset a_{l} \cup b_{l}$, it follows from the construction that $x \notin F_{l}$; hence $x \notin F$, 
which is a contradiction. This proves the claim, from which we now derive the lemma.

So let $l$ and $\left(a^{\prime}, b^{\prime}\right)$ be as in the claim. Then clearly $\left(a^{\prime}, b^{\prime}\right) \in \Sigma$ and $\left(a^{\prime}, b^{\prime}\right) \supset(a, b)$. Consider any $\left(a^{\prime \prime}, b^{\prime \prime}\right)$ in $\Sigma$ such that $\left(a^{\prime \prime}, b^{\prime \prime}\right) \supset\left(a^{\prime}, b^{\prime}\right)$. Set $a^{\prime \prime \prime}=a_{\mid A}^{\prime \prime}$ and $B=\operatorname{dom}\left(a^{\prime \prime}\right) \backslash A$. From the definition of $\Omega$ the set $B$ is finite and disjoint from the cylinder $\operatorname{dom}\left(b^{\prime \prime}\right)$, hence $C=(\pi(B) \times \omega) \backslash B$ is disjoint from the cylinder $\operatorname{dom}\left(a^{\prime \prime}\right) \cup \operatorname{dom}\left(b^{\prime \prime}\right)$ and we can define $b^{\prime \prime \prime}=b^{\prime \prime} \cup a^{\prime \prime} \cup \mathbb{1}_{\mid C}$. Since $\left(a^{\prime \prime \prime}, b^{\prime \prime \prime}\right) \in \Sigma^{\prime}$ and $\left(a^{\prime \prime \prime}, b^{\prime \prime \prime}\right) \supset\left(a^{\prime}, b^{\prime}\right)$, the claim yields $N_{a^{\prime \prime \prime} \cup b^{\prime \prime \prime}} \cap F_{l} \neq \emptyset$, and since $a^{\prime \prime} \cup b^{\prime \prime} \subset a^{\prime \prime \prime} \cup b^{\prime \prime \prime}$ we have $N_{a^{\prime \prime \prime} \cup b^{\prime \prime \prime}} \subset N_{a^{\prime \prime} \cup b^{\prime \prime}}$ and so $N_{a^{\prime \prime} \cup b^{\prime \prime}} \cap F_{l}$ $\neq \emptyset$.

Before we state the next lemma let us point out that our goal is to construct three sequences $\left(c_{n}^{(i)}\right)_{n \in \omega}$ for $i=0,1,2$ with similar properties. But for practical reasons we shall construct a unique sequence $\left(c_{n}\right)_{n \in \omega}$ and then define $c_{n}^{(i)}=c_{i+3 n}$.

Lemma 9.6. There exists a sequence $\left(\left(a_{n}, b_{n}\right), C_{n}, l_{n}\right)_{n \in \omega}$ in $\Sigma \times \mathcal{C} \times \omega$ such that denoting by $\equiv$ equality modulo 3 on the integers, and setting $A_{n}=$ $\bigcup_{k \equiv n, k<n} C_{k}$ and $B_{n}=\bigcup_{k \equiv n, k \leq n} C_{k}$, we have for all $n \geq 0$ :

(i) $C_{n} \cap \bigcup_{k<n} C_{k}=\emptyset$.

(ii) $\left(a_{n}, b_{n}\right) \supset\left(a_{n-3}, b_{n-3}\right)$.

(iii) $\operatorname{dom}\left(a_{n}\right) \subset A_{n}$ and $\operatorname{dom}\left(b_{n}\right)=B_{n}$.

(iv) $\operatorname{dom}\left(a_{n}\right) \supset A_{n}^{\prime}:=A_{n} \cap\left(\operatorname{dom}\left(a_{n-1}\right) \cup \operatorname{dom}\left(a_{n-2}\right)\right)$.

(v) $a_{n} . a_{n-1} \subset \mathbf{0}$ and $a_{n} . a_{n-2} \subset \mathbf{0}$.

(vi) For all $(a, b) \in \Sigma$ if $(a, b) \supset\left(a_{n}, b_{n}\right)$ then $N_{a \cup b} \cap G_{\left(n, l_{n}\right)} \neq \emptyset$.

(vii) $N_{a_{n} \cup b_{n}} \subset \bigcap_{k \equiv n, k, m<n} G_{\left(k, l_{k}, m\right)}$.

Here by convention $a_{k}=b_{k}=C_{k}=\emptyset$ for $k<0$.

Proof. Assume that $\left(\left(a_{k}, b_{k}\right), C_{k}, l_{k}\right)_{k<n}$ is constructed, and observe that the sets $A_{n}$ and $A_{n}^{\prime}$ are already determined. Choose a finite set $A$ such that $A_{n}^{\prime} \subset A \subset A_{n}$ and $\pi\left(A_{n}\right)=\pi(A)$, and consider $a=a_{n-3} \cup \mathbf{0}_{\mid A}$.

For all $k<n$ with $k \equiv n,\left(a, b_{n-3}\right) \in \Sigma$, and by (ii) $k$,

$$
\left(a, b_{n-3}\right) \supset\left(a_{n-3}, b_{n-3}\right) \supset\left(a_{k}, b_{k}\right) \text {. }
$$

It then follows from (vi) and Lemma 9.4 that each of the $\boldsymbol{\Pi}_{2}^{0}$ sets $G_{\left(k, l_{k}\right)}$ is dense in $N_{a \cup b_{n-3}}$. Hence $\bigcap_{k \equiv n, k<n} G_{\left(k, l_{k}\right)}$ is dense in $N_{a \cup b_{n-3}}$ and a fortiori so is the larger open set $\bigcap_{k \equiv n, k, m<n} G_{\left(k, l_{k}, m\right)}$, so we can find a finite extension $c$ of $a \cup b_{n-3}$ such that $N_{c} \subset \bigcap_{k \equiv n, k, m<n} G_{\left(k, l_{k}, m\right)}$. Then applying Lemma 9.5 to $\left(c \backslash b_{n-3}, b_{n-3}\right)$ we get $\left(a^{\prime}, b^{\prime}\right) \in \Sigma$ with $\left(a^{\prime}, b^{\prime}\right) \supset\left(c \backslash b_{n-3}, b_{n-3}\right) \supset$ $\left(a_{n-3}, b_{n-3}\right)$ such that for any $\left(a_{n}, b_{n}\right) \in \Sigma$ with $a_{n} \cup b_{n} \supset c^{\prime}:=a^{\prime} \cup b^{\prime}$ condition (vi) is satisfied.

Observe that since $\operatorname{dom}(a) \cap \operatorname{dom}\left(b^{\prime}\right)=\emptyset$ we have $\pi\left(A_{n}\right) \cap \pi\left(\operatorname{dom}\left(b^{\prime}\right)\right)=$ $\pi\left(A \cap \operatorname{dom}\left(b^{\prime}\right)\right)=\emptyset$, and consequently $A_{n} \cap \operatorname{dom}\left(b^{\prime}\right)=\emptyset$. 
Now set $C_{n}^{\prime}=\left(\pi\left(\operatorname{dom}\left(c^{\prime}\right)\right)\right) \times \omega, C_{n}=C_{n}^{\prime} \backslash \bigcup_{k<n} C_{k}, B_{n}=C_{n}^{\prime} \backslash A_{n}$ so that $B_{n}=\bigcup_{k \equiv n, k \leq n} C_{k}$ and define $a_{n}=c_{\mid A_{n}}^{\prime}=a_{\mid A_{n}}^{\prime}$ and $b_{n}=c_{\mid B_{n}}^{\prime} \cup \mathbb{1}_{\mid B_{n} \backslash \operatorname{dom}\left(c^{\prime}\right)}$. Then one can easily check that $\left(a_{n}, b_{n}\right) \in \Sigma$ and that conditions (i)-(vii) are all satisfied.

To finish the proof of Theorem 9.3 set $C=\omega^{2} \backslash \bigcup_{n \in \omega} C_{n}$ and define $x^{(i)}:=\bigcup_{k} a_{i+3 k} \cup b_{i+3 k} \cup \mathbf{0}_{\mid C}$ for $i=0,1$, 2. It follows from condition (vii) of Lemma 9.6 that each $x^{(i)}$ is in $G$, and we now check that $x^{(0)} \cdot x^{(1)} \cdot x^{(2)}(u)$ $=0$ for all $u \in \omega^{2}$. So fix such a $u$.

If $u \in C$ then by definition $x^{(i)}(u)=0$ for all $i$.

If not then by conditions (i) and (iii) there exists a unique pair $(i, k) \in$ $3 \times \omega$ such that $u \in \operatorname{dom}\left(b_{i+3 k}\right)$. Let $m<n$ be the first two integers $\not \equiv i$ such that $u \in \operatorname{dom}\left(a_{m}\right) \cap \operatorname{dom}\left(a_{n}\right)$. It follows from (iv) that $n=m+1$ or $m+2$, hence $m=n-1$ or $n-2$, so by $(\mathrm{v}), a_{m}(u) \cdot a_{n}(u)=0$. But since $m \neq \equiv n$, we have $a_{m} \subset x^{\left(i^{\prime}\right)}, a_{n} \subset x^{\left(i^{\prime \prime}\right)}$ with $\left\{i, i^{\prime}, i^{\prime \prime}\right\}=\{0,1,2\}$, and it follows that $x^{(0)} \cdot x^{(1)} \cdot x^{(2)}(u)=0$.

REMARKS 9.7. (a) We do not know of any direct proof of Theorem 9.3 avoiding the reduction argument of the general case to the particular case $\mathcal{F}=\mathcal{N}_{2}$. Observe in particular that the notion of cylinder, which played a crucial role in this proof, is meaningless in the general case, and the set $\mathcal{C}$ of all cylinders cannot be replaced by the ideal $\mathcal{F}^{*}$.

(b) The conclusion of Theorem 9.3 cannot be strengthened to yield two disjoint elements as in Theorem 9.2. Indeed, if

$$
\mathcal{G}=\left\{M \subset \omega^{2}: \exists p, M(p) \text { is cofinite and } \forall q \geq p, M(q) \text { is infinite }\right\}
$$

(where for all $M \subset \omega^{2}$ and $p \in \omega$ we set $M(p)=\{n \in \omega:(p, n) \in M\}$ ) then $\mathcal{G}$ is clearly a $\boldsymbol{\Sigma}_{3}^{0}$ set containing the filter $\mathcal{N}_{2}$ and one easily checks that any two elements of $\mathcal{G}$ have non-empty intersection.

\section{References}

[1] R. Baire, Sur les fonctions de variables réelles (Thèse), Ann. Mat. 3 (1899), 1-123.

[2] J. Calbrix, Filtres boréliens sur l'ensemble des entiers et espaces des applications continues, Rev. Roumaine Math. Pures Appl. 33 (1988), 655-661.

[3] T. Dobrowolski and W. Marciszewski, Classification of function spaces with the pointwise topology determined by a countable dense set, Fund. Math. 148 (1995), $35-62$.

[4] M. Katětov, Products of filters, Comm. Math. Univ. Carolin. 9 (1968), 173-184.

[5] - On descriptive classes of functions, in: Theory of Sets and Topology, Deutsch. Verlag Wiss., Berlin, 1972, 265-278.

[6] - On descriptive classification of functions, in: Proc. Third Prague Topological Symposium, Academia, Praha, 1972, 235-242.

[7] A. S. Kechris, Classical Descriptive Set Theory, Grad. Texts in Math. 156, Springer, New York, 1995. 
[8] H. Lebesgue, Sur les fonctions représentables analytiquement, J. Math. Pures Appl. 1 (1905), 139-216.

[9] A. Louveau, Sur la génération des fonctions boréliennes fortement affines sur un convexe compact métrisable, Ann. Inst. Fourier (Grenoble) 36 (1986), no. 2, 57-68.

[10] A. Louveau and J. Saint Raymond, Borel classes and closed games: Wadge-type and Hurewicz-type results, Trans. Amer. Math. Soc. 304 (1987), 431-467.

[11] —, - , The strength of Borel Wadge determinacy, in: Cabal Seminar 81-85, Lecture Notes in Math. 1333, Springer, 1988, 1-30.

[12] D. Lutzer, J. van Mill and R. Pol, Descriptive complexity of function spaces. Trans. Amer. Math. Soc. 291 (1985), 121-128.

[13] Y. N. Moschovakis, Descriptive Set Theory, North-Holland, Amsterdam, 1980.

[14] J. Saint Raymond, Fonctions boréliennes sur un quotient, Bull. Sci. Math. 100 (1976), 141-147.

[15] S. Solecki, Filters and sequences, Fund. Math. 163 (2000), 215-228.

Analyse Fonctionnelle

Institut de Mathématique de Jussieu

Boîte 186

4, place Jussieu

75252 Paris Cedex 05, France

E-mail: debs@math.jussieu.fr

raymond@math.jussieu.fr

Received 6 June 2008;

in revised form 18 May 2009 